\title{
RESEARCH
}

\section{Quantitative analysis of the mitochondrial proteome in human ovarian carcinomas}

\author{
Na Li ${ }^{1,2,3}$, Huanni Li4, Lanqin Cao4 and Xianquan Zhan ${ }^{1,2,3,5}$ \\ ${ }^{1}$ Key Laboratory of Cancer Proteomics of Chinese Ministry of Health, Xiangya Hospital, Central South University, Changsha, Hunan, People's Republic \\ of China \\ ${ }^{2 H u n a n}$ Engineering Laboratory for Structural Biology and Drug Design, Xiangya Hospital, Central South University, Changsha, Hunan, People's Republic \\ of China \\ ${ }^{3}$ State Local Joint Engineering Laboratory for Anticancer Drugs, Xiangya Hospital, Central South University, Changsha, Hunan, People's Republic of China \\ ${ }^{4}$ Department of Obstetrics and Gynecology, Xiangya Hospital, Central South University, Changsha, Hunan, People's Republic of China \\ ${ }^{5}$ The Laboratory of Medical Genetics, Central South University, Changsha, Hunan, People's Republic of China
}

Correspondence should be addressed to X Zhan: yjzhan2011@gmail.com

\begin{abstract}
Mitochondria play important roles in growth, signal transduction, division, tumorigenesis and energy metabolism in epithelial ovarian carcinomas (EOCS) without an effective biomarker. To investigate the proteomic profile of EOC mitochondrial proteins, a 6-plex isobaric tag for relative and absolute quantification (iTRAQ) proteomics was used to identify mitochondrial expressed proteins ( $m t E P s$ ) in EOCs relative to controls, followed by an integrative analysis of the identified mtEPs and the Cancer Genome Atlas (TCGA) data from 419 patients. A total of 5115 quantified proteins were identified from purified mitochondrial samples, and 262 proteins were significantly related to overall survival in EOC patients. Furthermore, 63 proteins were identified as potential biomarkers for the development of an EOC, and our findings were consistent with previous reports on a certain extent. Pathway network analysis identified 70 signaling pathways. Interestingly, the results demonstrated that cancer cells exhibited an increased dependence on mitophagy, such as peroxisome, phagosome, lysosome, valine, leucine and isoleucine degradation and fatty acid degradation pathways, which might play an important role in EOC invasion and metastasis. Five proteins (GLDC, PCK2, IDH2, CPT2 and HMGCS2) located in the mitochondrion and enriched pathways were selected for further analysis in an EOC cell line and tissues, and the results confirmed reliability of iTRAQ proteomics. These findings provide a large-scale mitochondrial proteomic profiling with quantitative information, a certain number of potential protein biomarkers and a novel vision in the mitophagy bio-mechanism of a human ovarian carcinoma.
\end{abstract}
Key Words
- ovarian carcinoma
- mitochondrial proteomics
- ITRAQ
- TCGA
- mitophagy
- biomarker

\section{Introduction}

Epithelial ovarian carcinoma (EOC) is the cause of more deaths than any other female genital tract cancers, and nearly accounts for $6 \%$ of all cancers among women (Sakhuja et al. 2017). Detection of early-stage EOCs is challenging since this disease is clinically asymptomatic in early stage, with being progressing toward the development of metastasis (Gadducci et al. 2007). In China, an increasing trend in mortality was observed for three of the ten most common cancers (breast, cervix and ovary), with stable trends for colorectum, lung, uterine 
and thyroid cancers (Chen et al. 2016). Even through tumor biomarkers CA125 and HE4 were widely used in clinical practice (Cymbaluk-Ploska et al. 2018), and more combined determinations would result in significantly improved sensitivity and efficiency, which might lead successfully to achieve a significant reduction in mortality.

Mitochondria are involved in various cellular processes, from regulation of metabolic flux to apoptosis. Mitochondrial dysfunctions have been proposed as a cause of cancer, are a biomarker for the early-stage detection of a cancer and are a therapeutic target for a cancer (Kim et al. 2017). Moreover, those mitochondrial ribosomal proteinencoding genes could be anti-oncogenes, which can even be new therapeutic targets or prognostic biomarkers. MRPL41, known as bcl-2-interacting mitochondrial ribosomal protein L41, indicated that the differential expression of MRPL41 in carcinomas is reflected by the various epigenetic states together with different responses and promoter methylation through the estrogen receptor (Kim et al. 2013). In addition, mitochondria could be 'fuel' to a cancer metabolism. Higher expression of MRPS15 in epithelial breast cells was revealed in an analysis of paired adjacent stromal tissues and neoplasm tissues (Sotgia et al. 2012). Those studies showed that the neoplasm-relevant communication pathways were linked with mitochondrial proteins. The role of mitochondrial ribosomal protein S23 (MRPS23) in carcinoma cell proliferation could be a potential therapeutic target, in case of interference of hepatocellular cancer proliferation, oncogenesis and metastasis (Pu et al. 2017). The expression of COX1 was involved in endometrial cancers (Ksiezakowska-Lakoma et al. 2017), esophageal adenocarcinoma (Huhta et al. 2017) and lung cancer (Michalak et al. 2016). Taken all together, these examples support the notion that mitochondria contribute to the angiogenetic, tumorigenic, proliferation, invasive and metastatic features of cancer cells. However, no large-scale quantitative reference map of a human EOC mitochondrial proteome was reported previously. Moreover, only focusing on single molecule biomarker is a narrowest form for cancer prediction, prevention and treatment (Cheng \& Zhan 2017, Zhan et al. 2017a). Cancer, by definition, is a kind of gene disease or protein disease, and results in a series of molecular alterations (GonzalezAngulo et al. 2012). Multiple biomarkers could provide a novel approach to predict, prevent and personalize the treatment of an EOC. Furthermore, newly discovered proteins could be used as potential targets or biomarkers (Wang et al. 2015). However, the proteomic profile of EOC mitochondrial proteins has not been elucidated. It is necessary to find a series of mitochondrial biomarkers on EOC in terms of detection technology. Proteomics has developed as a powerful approach to investigate novel biomarkers and drug targets (Ray et al. 2011), and it has mainly applied in the identification and quantification of proteomic components including post-translational modifications (Zhan et al. 2017b,c). Due to great improvement in mass spectrometry (MS) analysis, peptide identification and protein sequence coverage showed a preferable consistency in complex samples (Riley \& Coon 2018). The commonly used quantitative proteomic methods include gel-based such as 2D gel electrophoresis (2DGE) and 2D difference in-gel electrophoresis (2D DIGE) and gel-free-based (Hu et al. 2013), to allow highly sensitive and high-throughput identification of proteins/peptides and post-translational modifications (van der Wal et al. 2018). In general, gel-free methods are able to break through restrictions of gel-based methods that are inefficient in resolving proteins that are insoluble, lowly abundant or large proteins (>200 kDa) (Pasing et al. 2017). Multi-dimensional liquid chromatography-tandem mass spectrometry (MDLC-MS/MS)-based proteomics techniques were developed rapidly, and isobaric tag for relative and absolute quantification (iTRAQ) was featured with the advantages of strong separation ability and analysis range. A few of the advantages of the use of iTRAQ to reveal biomarkers or molecular mechanisms of different cancers have been reported (Zhou et al. 2017). Even 2DGE-MS with isotopic labeling and the application of high-sensitivity MS enables the quantification of a much larger part (estimated up to at least 500,000 protein species) of the human proteome as assumed before (Zhan et al. 2018).

This study used iTRAQ proteomics to identify and quantify mitochondrial expressed proteins (mtEPs) in EOCs. A total of 5115 proteins was identified and quantified. Moreover, Kyoto encyclopedia of genes and genomes (KEGG) analysis found a variety of signaling pathways such as peroxisome, phagosome, lysosome, valine, leucine and isoleucine degradation, and fatty acid degradation pathway, and they might play an important role in EOC mitophagy. The Cancer Genome Atlas (TCGA) database and mtEPs data were integrated and analyzed to investigate the gene ontology (GO) functional enrichments, protein-protein interactions and gene coexpression. Thus, 63 proteins as potential biomarkers for the development of EOCs were identified; and ERBB2, PTBP1 and H2AFX as biomarkers confirmed with previous reports were significantly related to overall survival in EOCs. These findings provide a certain number of proteins with the potential biomarkers and a novel vision 
in the mitophagy bio-mechanism of human EOCs. More importantly, we propose the use of the multi-biomarkers strategy to predict, prevent and personalize the treatment of an EOC.

\section{Materials and methods}

\section{EOC tissue specimen}

Ovarian tissues provided by Department of Gynecology, Xiangya Hospital (Changsha, China) were obtained from 18 female patients (EOC: $n=7$ and control: $n=11$ ) and were approved by the Xiangya Hospital Medical Ethics Committee of Central South University, and informed consent was obtained from the participation. Seven EOC patients were diagnosed as high-grade, poorly or moderately differentiated carcinoma cells. Eleven control ovaries were with benign gynecologic diseases, such as fibroids, adenomyosis, ovary serous cystadenoma, cervical intraepithelial neoplasia, atypical hyperplasia of endometrium and pelvic organ prolapse. Each obtained tissue was quickly put into liquid nitrogen and then stored in $-80^{\circ} \mathrm{C}$.

\section{Preparation of mitochondria}

Ovarian tissue samples were divided into EOC $(n=7)$ and control $(n=11)$ groups. The mitochondrial isolation buffer was prepared with $210 \mathrm{mM}$ mannitol, $70 \mathrm{mM}$ sucrose, $100 \mathrm{mM}$ potassium chloride $(\mathrm{KCl}), 1 \mathrm{mM}$ diamine tetraacetic acid (EDTA), $50 \mathrm{mM}$ Tris- $\mathrm{HCl}, 0.1 \mathrm{mM}$ ethylene glycol bis(2-aminoethyl ether)tetraacetic acid (EGTA), $1 \mathrm{mM}$ phenylmethanesulfonyl fluoride (PMSF) protease inhibitor, $2 \mathrm{mM}$ sodium orthovanadate (V), $0.2 \%$ bovine serum albumin (BSA) and $\mathrm{pH}$ 7.4. (i) The EOC tissues $(1.5 \mathrm{~g})$ were fully minced $\left(1 \mathrm{~mm}^{3}\right.$ pieces $)$ and homogenized $\left(2 \mathrm{~min} ; 4^{\circ} \mathrm{C}\right)$ in $13.5 \mathrm{~mL}$ mitochondrial isolation buffer that contained $0.2 \mathrm{mg} / \mathrm{mL}$ Nagarse. The tissue homogenates were well mixed with another $3 \mathrm{~mL}$ mitochondrial isolation buffer, followed by centrifugation $\left(1300 \boldsymbol{g}, 10 \mathrm{~min}\right.$ and $\left.4^{\circ} \mathrm{C}\right)$ to remove crude nuclear fraction. The supernatant was re-centrifuged $(10,000 \boldsymbol{g}, 10 \mathrm{~min}$ and $4^{\circ} \mathrm{C}$ ) to remove microsomes in the supernatant. The debris was re-suspended in $2 \mathrm{~mL}$ mitochondrial isolation buffer, followed by centrifugation $\left(7000 \mathrm{~g}, 10 \mathrm{~min}\right.$ and $4^{\circ} \mathrm{C}$ ) to obtain crude mitochondria in the debris (Jiang et al. 2004). The extracted crude mitochondria were dissolved in $12 \mathrm{~mL} 25 \%$ Nycodenz (Sigma), and then a discontinuous Nycodenz gradient was made through filling with $5 \mathrm{~mL}$ of $34 \%, 8 \mathrm{~mL}$ of $30 \%, 12 \mathrm{~mL}$ of $25 \%$ (contained crude mitochondria), $8 \mathrm{~mL}$ of $23 \%$, and $3 \mathrm{~mL}$ of $20 \%$ Nycodenz from bottom to top in a tube, followed by centrifugation $\left(52,000 \mathrm{~g}, 90 \mathrm{~min}, 4^{\circ} \mathrm{C}\right)$ (Schonenberger \& Kovacs 2017). After centrifugation, the purified mitochondria were contained at the interface of $25-30 \%$, which was collected in a clean tube. The collected mitochondria were diluted with mitochondrial isolation buffer to three-fold volume, and centrifuged $\left(15,000 \mathrm{~g}, 20 \mathrm{~min}, 4^{\circ} \mathrm{C}\right)$. The debris was re-suspended in $2 \mathrm{~mL}$ mitochondrial isolation buffer, followed by centrifugation $\left(15,000 \mathrm{~g}, 20 \mathrm{~min}, 4^{\circ} \mathrm{C}\right)$. The final debris was the purified mitochondria. (ii) For the mitochondria purified from the control tissues, the above procedure (i) was modified as the following: prior to homogenization, $8 \mathrm{~mL}$ of $0.05 \%$ trypsin $/ 20 \mathrm{mM}$ EDTA in PBS solution was added to the minced control tissues and digested for $30 \mathrm{~min}$ at room temperature, followed by centrifugation ( $200 \mathrm{~g}, 5 \mathrm{~min}$ ). The discontinuous Nycodenz gradient was made through filling with $8 \mathrm{~mL}$ of $38 \%, 5 \mathrm{~mL}$ of $34 \%, 8 \mathrm{~mL}$ of $30 \%, 12 \mathrm{~mL}$ of $25 \%$ (contained crude mitochondria), $8 \mathrm{~mL}$ of $23 \%$ and $3 \mathrm{~mL}$ of $20 \%$ Nycodenz from bottom to top in a tube, followed by centrifugation $\left(52,000 \mathrm{~g}, 90 \mathrm{~min}, 4^{\circ} \mathrm{C}\right)$. After centrifugation, the purified mitochondria were contained at the interface of $25-30 \%$ to the interface of $34-38 \%$. The other procedure is the same as the procedure (i).

All purified mitochondria from EOC and control tissues were put together, respectively. Then, proteins were extracted from purified EOC and control mitochondria, respectively.

\section{ITRAQ quantitative proteomics and bioinformatics}

The purified EOC and control mitochondria were digested with trypsin. (i) iTRAQ labeling: The tryptic peptides were labeled with a 6-plex iTRAQ Multiplex Kit according to the manufacturer's instructions (Applied Biosystems iTRAQ Reagents-Chemistry Reference Guide, P/N 4351918A). Briefly, the peptides were dissolved in $100 \mathrm{mM}$ tetraethyl ammonium bromide solution ( $\mathrm{pH}$ 8.5) before the labeling reagent was added. After 2-h incubation, the reaction was quenched by adding an equal volume of water. Six labeled peptide samples were mixed equally and dried with a speed-vac (Qi et al. 2016). (ii) Strong cation exchange (SCX) fractionation: The labeled peptide mixture was fractionated with SCX chromatography. (iii) LC-MS/MS: Each fractionation was subjected to LC-MS/MS analysis on a Q Exactive mass spectrometer (Thermo Scientific) that was coupled to Easy nLC (Proxeon Biosystems, now Thermo Fisher Scientific) for $60 \mathrm{~min}$. MS/MS spectra were acquired and used to search protein database with 
MASCOT engine (Matrix Science, London, UK; version 2.2) embedded into Proteome Discoverer 1.4. The identified proteins were used for KEGG pathway analysis with Cytoscape (two-sided hypergeometric test, Kappa score $>0.9$, adjusted $P$ value $<0.01$ corrected with BenjaminiHochberg), and further for KEGG pathway enrichment with DAVID Bioinformatics Resources 6.7 (https:// david.ncifcrf.gov/home.jsp). Heat map was plotted by Multiple Experiment Viewer (https://sourceforge.net/ projects/mev-tm4/files/mev-tm4/). GO biological process (BP), molecular function (MF) and cellular component (CC) were analyzed with Cytoscape ClueGO (two-sided hypergeometric test, adjusted $P$ value $<0.05$ corrected with Benjamini-Hochberg). GO CC was further enriched with PANTHER (http://www.pantherdb.org/). Biomarkers that had been reported were checked by CooLGeN (http:// ci.smu.edu.cn/CooLGeN/Home.php).

\section{TCGA data of EOC patients}

TCGA data portal provides a platform for researchers to search, download and analyze datasets generated from TCGA database (http://cancergenome.nih.gov/) (Zhang et al. 2016). Level 3 RNA-seq V2 and clinical data were obtained from the TCGA data of 419 EOC patients. The gene expression missing value (expression=0) more than $20 \%$ was excluded after the pretreatment. Overall survival analysis of genes in EOCs was calculated by the Kaplan-Meier method, and compared to the log-rank test with R 3.4.2 version (https://www.r-project.org/). The $P$ value less than 0.01 was considered as statistically significant.

\section{Prediction of protein-protein interaction}

The overlapped proteins of iTRAQ-identified proteins and TCGA overall related genes were analyzed by STRING 10.0 (http://string-db.org/cgi/input.pl) with a high confidence of parameter $(>0.700)$.

\section{Construction of mRNA-mRNA network of the overlapped proteins}

The Pearson correlation test was used to determine whether the expression levels of overlapped proteins of ITRAQ identified proteins and TCGA overall related genes were correlated with each other, respectively. The mRNA-mRNA correlations of those overlapped proteins with $P$ values $<0.05$ were corrected with BenjaminiHochberg. Extracted data with negative correlation $(r<-0.5)$ or positive correlation $(r>0.7)$ from were used to construct circus chart by Package RCircos. Each line denoted one mRNA-mRNA pair. Each region along the circle represented one of the 24 chromosomes (Zhang et al. 2013). Chromosomal location was obtained by Ensembl (http://asia.ensembl.org/index.html).

\section{Cell lines and cell culture}

EOC cell line TOV-21G cells and control cell line IOSE80 cells were purchased from Keibai Academy of Science (Nanjing, China). TOV-21G cells were cultured in RPMI-1640 medium, and IOSE80 cells were cultured in DMEM medium (Corning, NY, USA) supplemented with $10 \%$ fetal bovine serum (FBS, Gibco). All these cells were maintained with $5 \% \mathrm{CO}_{2}$ atmosphere at $37^{\circ} \mathrm{C}$.

\section{RNA extraction and quantitative real-time PCR (qRT-PCR) analyses}

Total RNAs were extracted from cell lines with TRizol Reagent (Invitrogen) according to the manufacturer's instructions. For the detection of SNHG3 and target genes, total RNAs were reversely transcribed into cDNAs and then used to perform qRT-PCR with SYBR Premix Ex Taq (TaKaRa). $\beta$-actin was used as an internal control for mRNA quantification.

\section{Western blotting}

Equal amounts of proteins were separated by $10 \%$ SDS-PAGE gels and blotted onto nitrocellulose membranes. The blotted proteins on the membrane were incubated with primary antibodies against GLDC, PCK2, IDH2, CPT2 and HMGCS2 (1:1000; Abcam) and $\beta$-actin (1:2000; Santa Cruz Biotechnology) at $4^{\circ} \mathrm{C}$ overnight. The membranes were incubated for $2 \mathrm{~h}$ with horseradish peroxidase-conjugated goat anti-rat secondary antibody (1:5000; Santa Cruz Biotechnology) at room temperature.

\section{Statistical analysis}

Data were expressed as the mean \pm s.D. of triplicates. Each experiment was repeated at least three times. Statistical analyses were performed using SPSS 13.0 (SPSS Inc.). The Student's t-test was used to assess the between-group differences of in vitro studies with a statistical significance level of $P<0.05$. Some cases were corrected with BenjaminiHochberg (FDR) for multiple testing. 
A

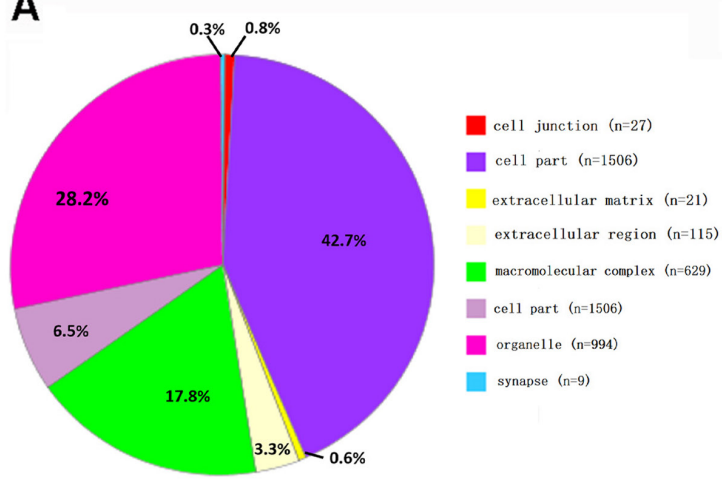

C

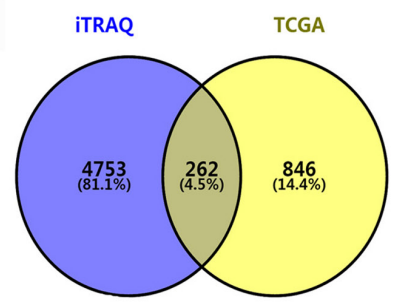

D

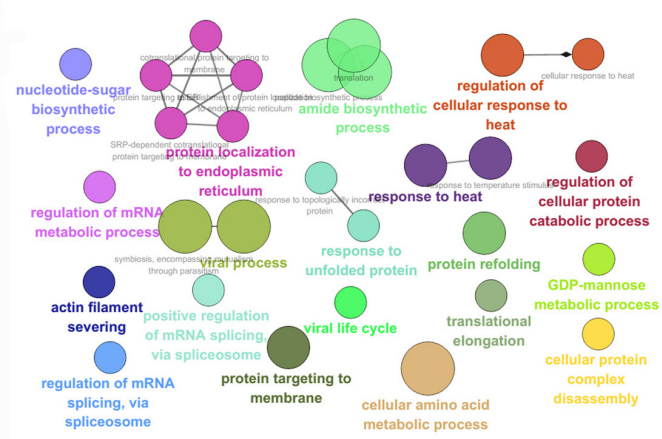

E

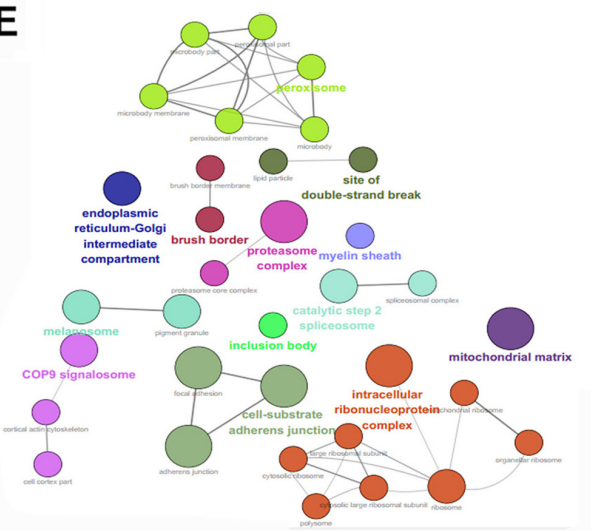

B

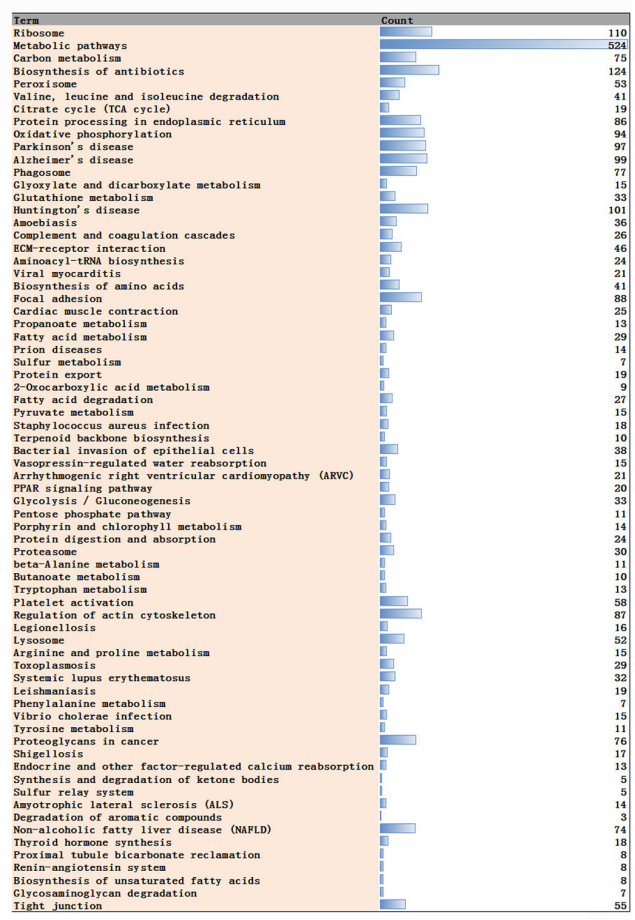

$\mathbf{F}$

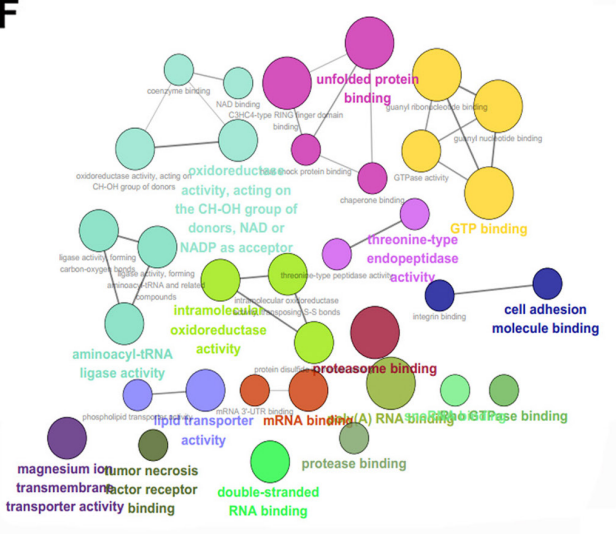

Figure 1

Network analysis of identified proteins by iTRAQ. (A) A total of 5115 proteins was classified according to the cell components with PANTHER. (B) KEGG pathway analysis mapped the identified proteins to 70 signaling pathways. (C). The overlapped proteins were obtained between 5115 identified proteins and TCGA overall related survival genes. (D, E and F) The overlapped proteins were classified according to the biological process (BP), cellular component (CC) and molecular function (MF). The less $P$ value and more significant enrichment were shown with the greater node size. The same color indicated the same function group. Among the groups, we chose a representative of the most significant term and lag highlighted. A full colour version of this figure is available at https://doi.org/10.1530/ERC-18-0243. 


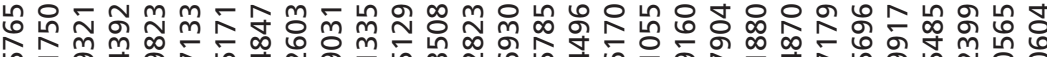

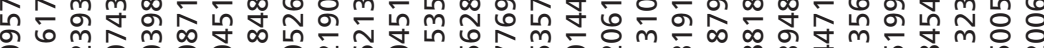

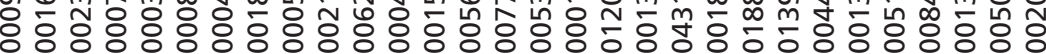

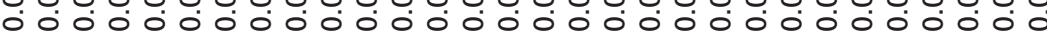

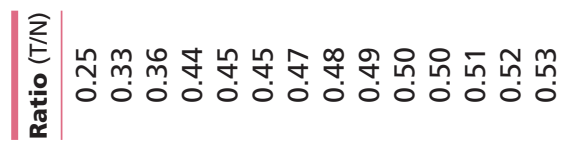

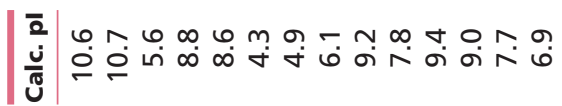
|ึิ m

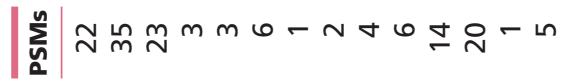

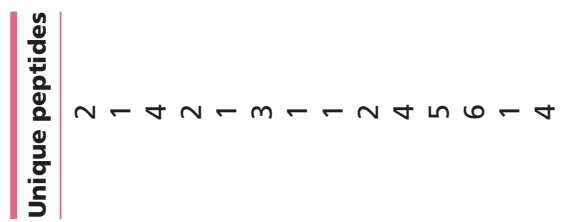

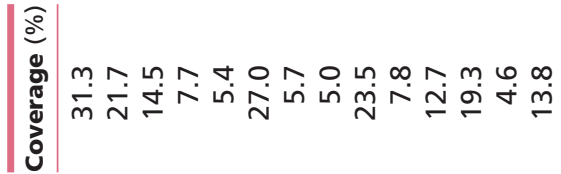

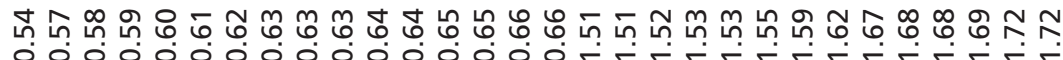

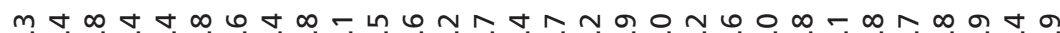
ढ बं

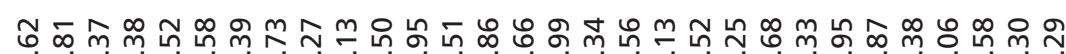
ஸ்

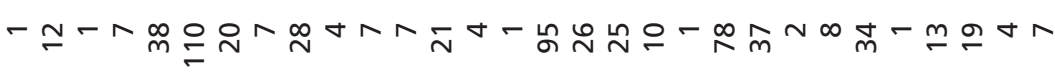

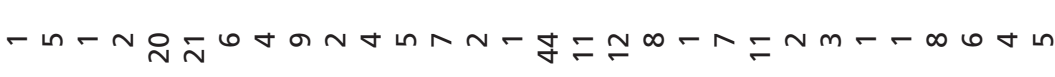

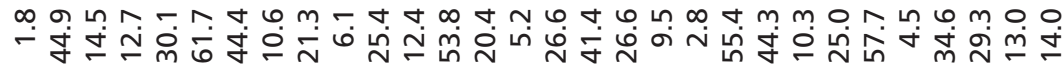

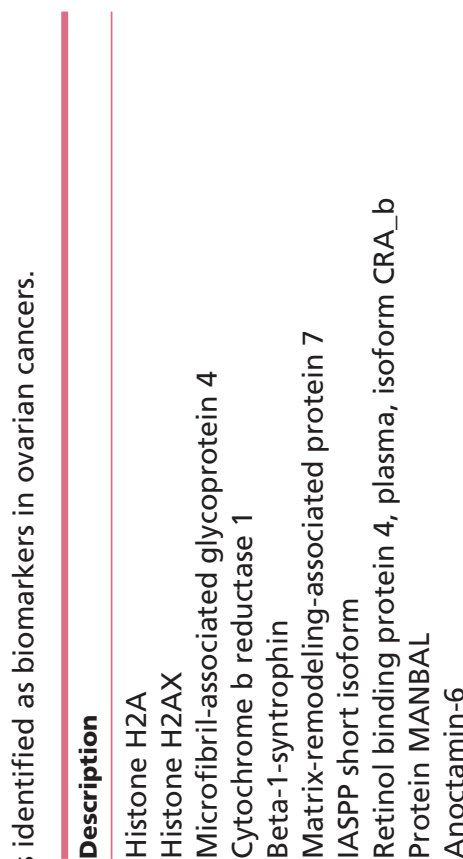

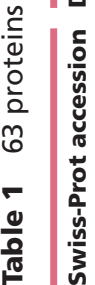

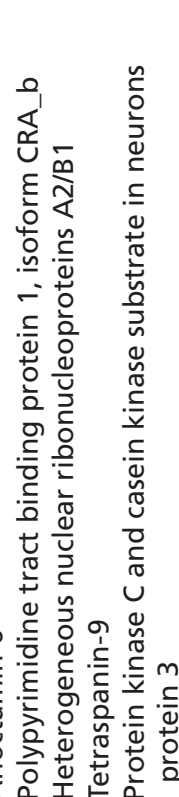

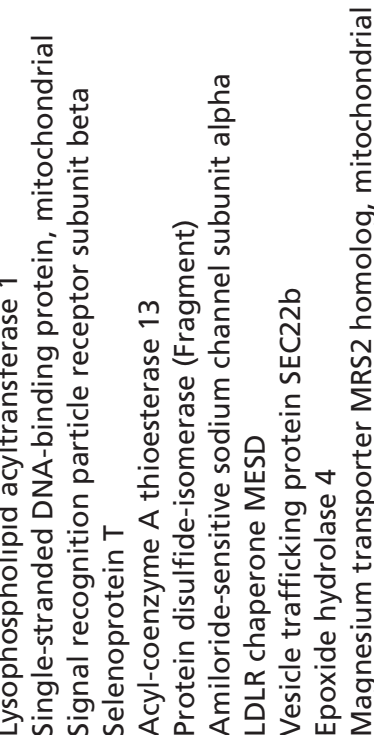

$\sum_{3}^{\bar{c}}$

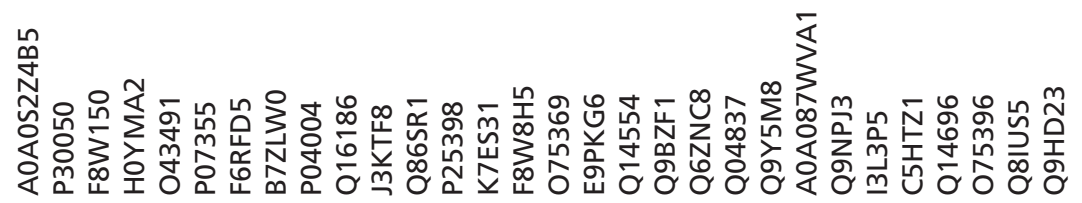




\section{Results}

\section{Quantitative proteome map of EOC mitochondria}

The iTRAQ-SCX-LC-MS/MS quantitative analysis of isolated mitochondrial samples identified a total of 5115 proteins that were present in EOC and control tissues (Supplementary Table 1, see section on supplementary data given at the end of this article). Each protein was identified with at least one peptide sequence matches (PSMs). Those proteins identified in EOC and control tissues were distributed within a range of molecular weight (MW) 2.6-1158.2 kDa and pI 3.81-12.25, which was consistent with the pI distribution pattern of the previously identified proteins in the mitochondrial fraction reported by Rezaul et al. (Rezaul et al. 2005). In addition, iTRAQ proteomic analysis also obtained protein quantitative information, including 2565 (50.14\%) upregulated proteins and 2550 (49.86\%) downregulated proteins in EOC relative to control tissues. The top 10 upregulated proteins were androgeninduced 1/Golgi SNAP receptor complex member 1 variant 1 fusion protein (AIG1), pituitary tumor-transforming gene 1 protein-interacting protein (PTTG1IP), protein S100-A14 (S100A14), agmatinase mitochondrial (AGMAT), normal mucosa of esophagus-specific gene 1 protein (NMES1), tetraspanin-1 (TSPAN1), protein-glutamine gammaglutamyltransferase K (Fragment) (TGM1), MHC class II antigen (Fragment) (HLA-DPA1), uncharacterized protein C1orf53 (fragment) (C1orf53) and MHC class I antigen (Fragment) (HLA-A). The top 10 downregulated proteins were collagen alpha-1(II) chain (COL2A1), nanospan (NSPN), SUN domain-containing protein 2 (fragment) (SUN2), pumilio homolog 1 (Fragment) (PUM1), ubiquitin C (UbC), Xin actin-binding repeat-containing protein 1 (XIRP1), cytochrome c oxidase subunit 4 isoform 2 (COX4I2), histone H2A type 2-B (HIST2H2AB), collagen, type V, alpha 2, isoform CRA_b (COL5A2) and collagen alpha-1 (X) chain (COL10A1). Comparative proteomics analysis focuses on those statistically significantly differentially expressed proteins (DEPs) that might be the real potential biomarkers. However, one could not ignore those no-expression-level-changed proteins, which might have experienced post-translational modifications, such as phosphorylation, glycosylation, acetylation, methylation, nitration, ubiquitylation, sumoylation, succinylation, sulfation, myristoylation, palmitoylation, deamidation, prenylation and hydroxylation (Cleland 2018, Khan et al. 2018, Sloutsky \& Naegle 2018) to result in different proteoforms or they might be hubmolecules in a molecular network with less changes 
A

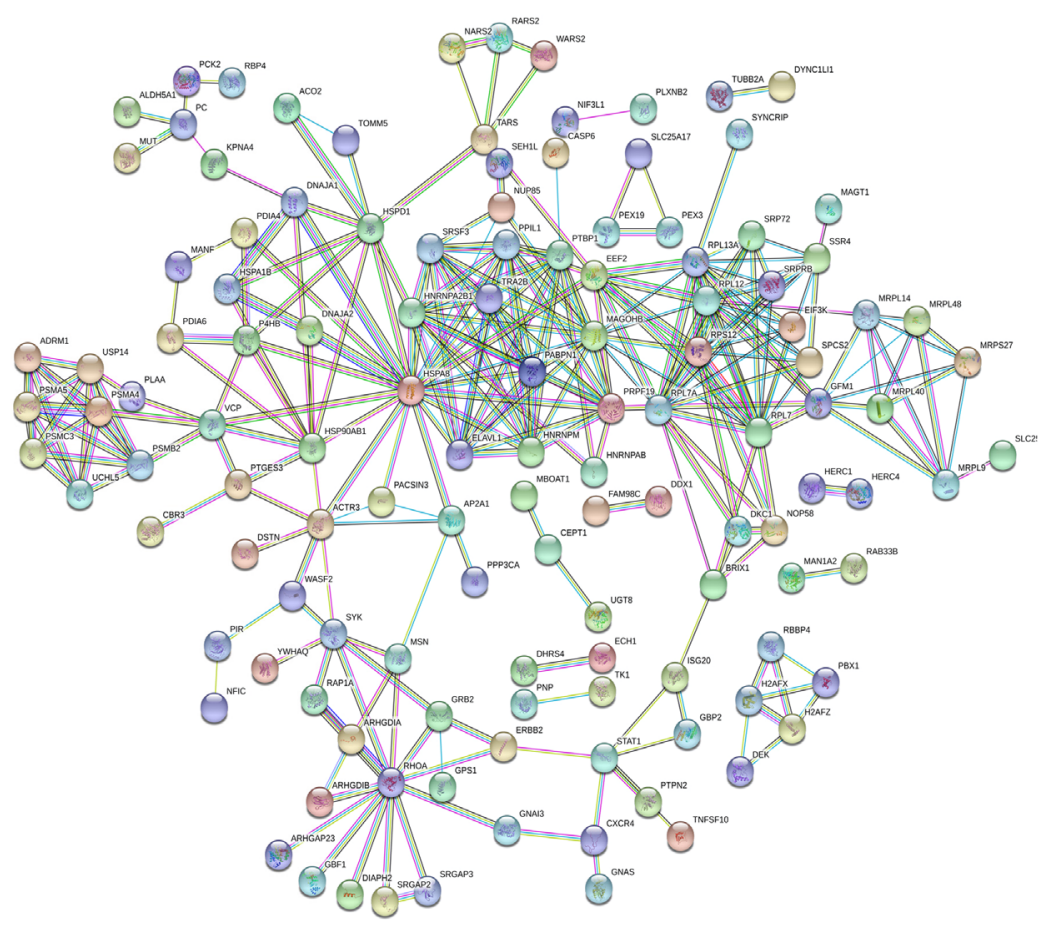

D

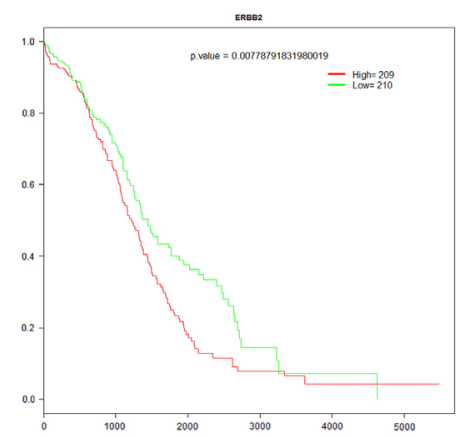

E

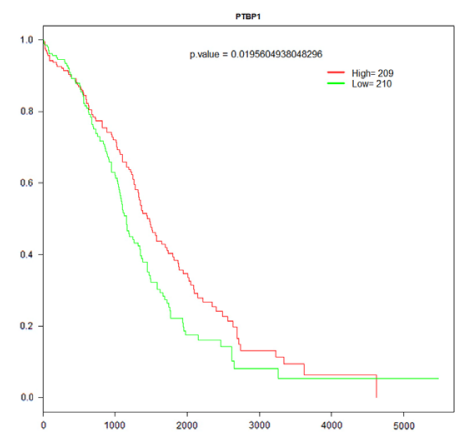

B

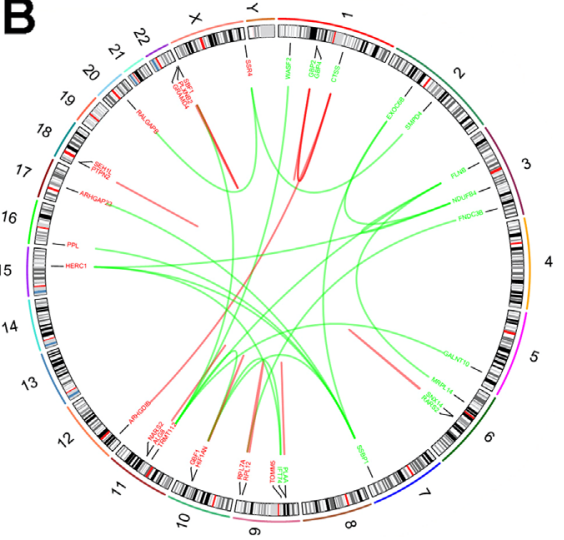

C

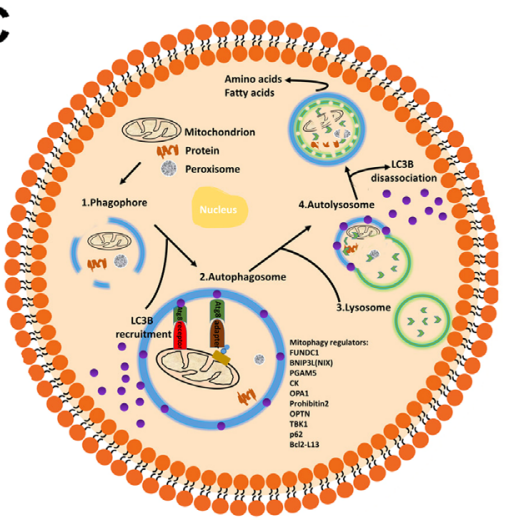

F

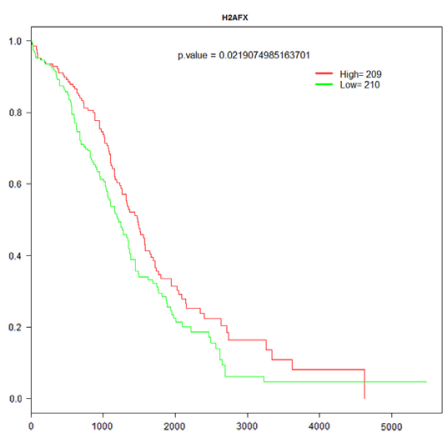

Figure 2

The interaction networks of identified proteins. (A) The protein-protein interactions (PPIs) network of 262 overlapped proteins. (B) The mRNA-mRNA pair analysis of 262 overlapped proteins constructed circus chart by R package. Upregulated proteins by iTRAQ in red letters and downregulated proteins in green letters. Red line represents positive correlation and green line represents negative correlation. (C) Cancer cells exhibit an increased dependence on mitophagy, such as peroxisome, phagosome, valine, leucine and isoleucine degradation, fatty acid degradation pathway. D-F. Kaplan-Meier (KM) survival curve of ERBB2, PTBP1 and H2AFX in an epithelial ovarian carcinoma (EOC). A full colour version of this figure is available at https://doi. org/10.1530/ERC-18-0243.

relative to the boundary molecules in a given condition (Zhan et al. 2017b).

\section{GO enrichment analysis}

To ascertain how the identified proteins promoted phenotype in an EOC tissue, GO analysis was performed using PANTHER. A total of 5115 proteins were classified according to the CCs. Figure $1 \mathrm{~A}$ showed variable CCs: cell part (42.7\%), organelle (28.2\%) and macromolecular complex (17.8\%). Moreover, 1108 overall survival-related genes were obtained by TCGA database (Supplementary Tables 2, 3 and 4). There were 262 significant proteins (Supplementary Table 5) obtained when overlapping 

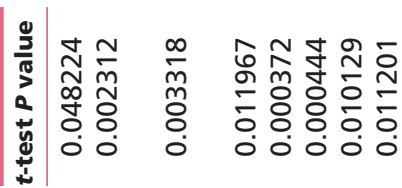

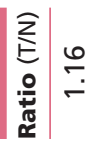

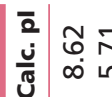

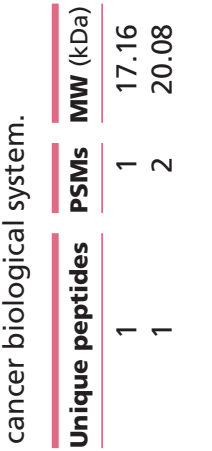

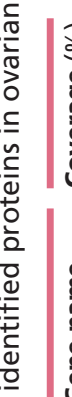

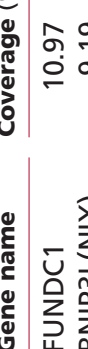

กำ ᄂ

ભึ

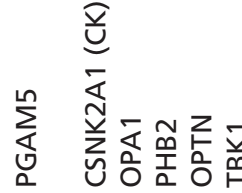

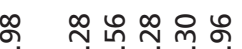

宛宅白㐫

$\hat{m}$

$\infty \stackrel{m}{\stackrel{N}{N}}$

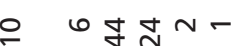

을
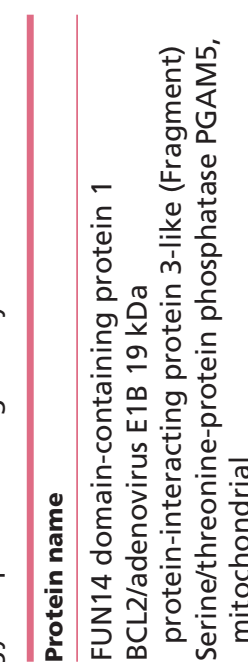
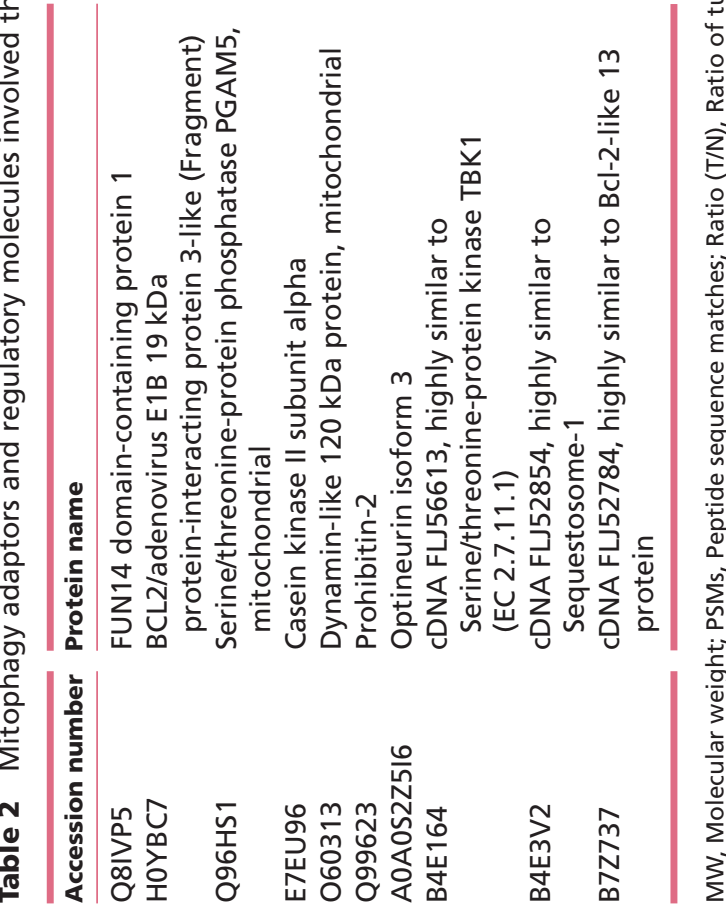

analysis was performed between 5115 identified proteins and TCGA database (Fig. 1C). Moreover, 262 overlapped proteins were classified according to the BP, CC and MF. As shown in Fig. 1D, E and F, the less $P$ value and more significant enrichment were shown with the greater node size. The same color indicated the same function group. Among the groups, a representative was chosen of the most significant term and lag highlighted. The overlapped proteins were mainly distributed in endoplasmic reticulum, cellular amino acid metabolic process, viral process, regulation of cellular response to heat and response to heat according to BP (Supplementary Table 6). The localization of overlapped proteins was also differentially distributed in peroxisome, COP9 signalosome, intracellular ribonucleoprotein complex, cell-substrate adherens junction and mitochondrial matrix according to CC (Supplementary Table 7). A series of MFs were involved in 262 overlapped proteins, including GTP binding, unfolded protein binding, oxidoreductase activity (acting on $\mathrm{CH}-\mathrm{OH}$ group of donors), intramolecular oxidoreductase activity and aminoacyl-tRNA ligase activity (Supplementary Table 8). Compared to previous studies, 992 proteins of 5115 proteins have been reported to relate with ovary (Supplementary Table 9). For example, VTCN1 was overexpressed in early-stage EOCs and was independent of CA125 expression (Simon et al. 2007, Fortner et al. 2018) and CDKN2A was the similar biomarker for early-stage EOCs (Jiang et al. 2017). CD44, PLAT and PTBP1 showed prognostic value in EOC patients (Borgfeldt et al. 2003, Zhang et al. 2010, Bartakova et al. 2018). Correlation between tumor mesothelin (MSLN) expression and serum MSLN in EOC patients was fine (Hanaoka et al. 2017). In our findings, 63 proteins were identified as novel biomarkers in EOCs (the fold change $\geq 1.5$ ) (Table 1). Our findings were consistent with previous reports on a certain extent such that ERBB2, PTBP1 and H2AFX were not only biomarkers but also significantly related to overall survival (Fig. 2D, E and F).

\section{The protein-protein and mRNA-mRNA interactions of 262 overlapped proteins}

The 262 overlapped proteins were uploaded to STRING for protein-protein interaction analysis. The combined scores of nodes ranged from 0.700 to 0.999 . Some key proteins were identified in the part of the common proteins between 5115 identified proteins and TCGA database's RNAs, such as VCP (fold change $=1.04, P=0.05$ ), RHOA (fold change $=0.88, P=0.04$ ), RPL7A (fold change $=1.12$, $P=0.03$ ), HSPA8 (fold change $=0.90, \quad P=0.08$ ), 
HSPD1 (fold change $=1.42, \quad P=0.03$ ) and PRPF19 (fold change $=0.93, P=0.31$ ) (Fig. 2A; Supplementary Table 10). The 262 overlapped proteins were also used to construct circus chart by R package. Examination of the genomic location of the correlated mRNAs within each mRNA-mRNA pair revealed that most of these genes resided on different chromosomes. However, there were some gene pairs sited in different chromosomal location, including CTSS-GBP2, CTSS-GBP4, IFT74-PLAA, GRAMD4-SBF1, ALG8-NARS2， RPL12-RPL7A， GBP2GBP4, GBF1-HIF1AN, PLXNB2-SBF1, RARS2-SNX14 and PTPN2-SEH1L (Fig. 2B). These results indicated whether genes affected by each other through neighboring genes or not (Blinka et al. 2016). The mRNA-mRNA correlations were revealed (Supplementary Table 11), and chromosomal locations were obtained by Ensembl (Supplementary Table 12).

\section{KEGG pathway enrichment analysis indicated mitophagy}

KEGG pathway analysis mapped the identified proteins to 70 signaling pathways (Fig. 1B; Supplementary Table 13). Interestingly, the results demonstrated that cancer cells exhibited an increased dependence on mitophagy, such as peroxisome, phagosome, lysosome, valine, leucine and isoleucine degradation and fatty acid degradation pathway, which might play an important role in EOC

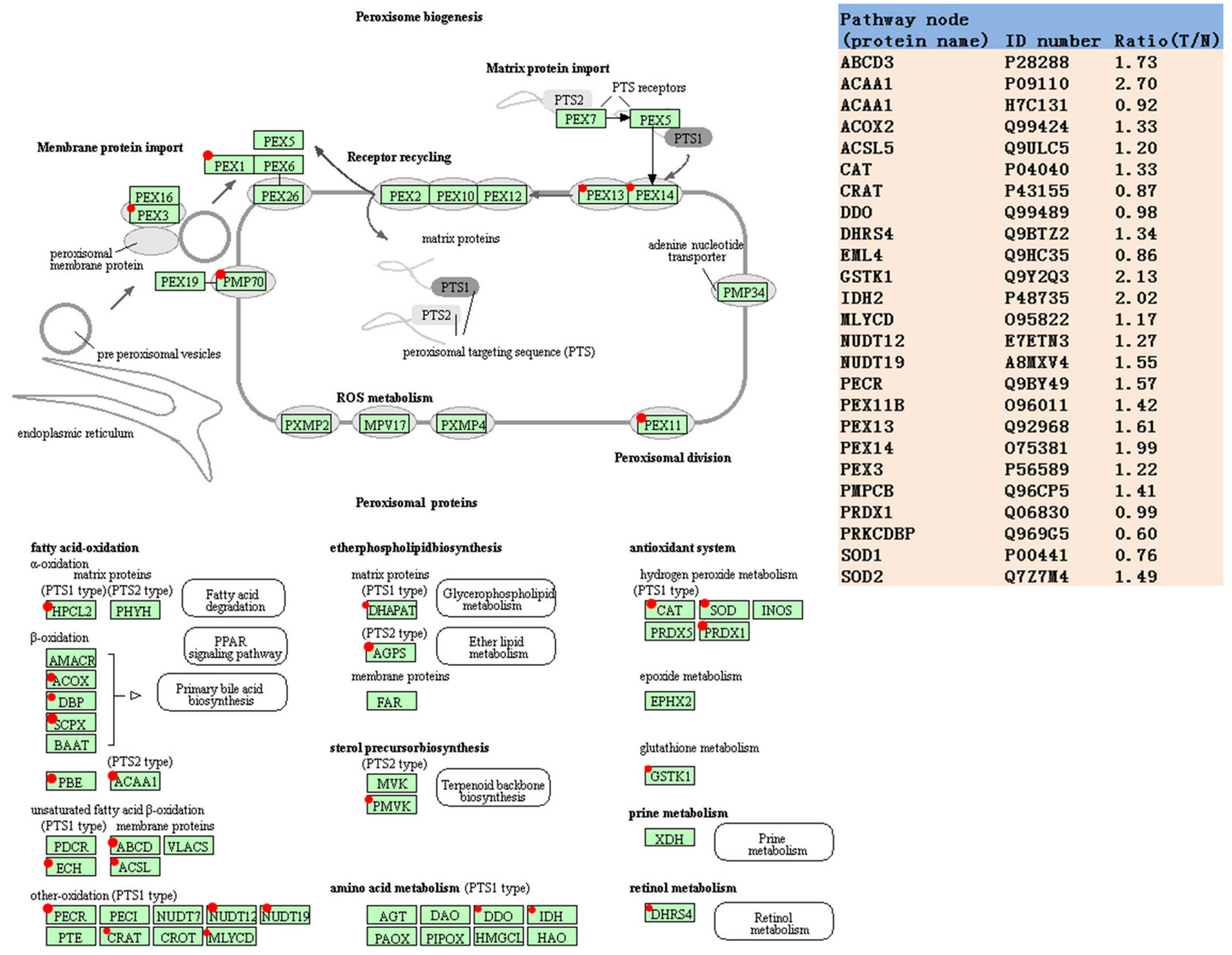

Figure 3

Peroxisome pathway altered in an ovarian cancer. Green rectangle with red mark means the identified proteins. Green rectangle without red mark means species-specific enzymes. White rectangle means reference pathway. The solid line means molecular interaction. The dot line means indirect effect. The circle means mostly chemical complex. The pathway node in the right panel corresponds to the red marked node in the left diagram. ID number is the Swiss-Prot accession number. Ratio $(\mathrm{T} / \mathrm{N})=$ Ratio of tumors to controls. A full colour version of this figure is available at https://doi. org/10.1530/ERC-18-0243. 


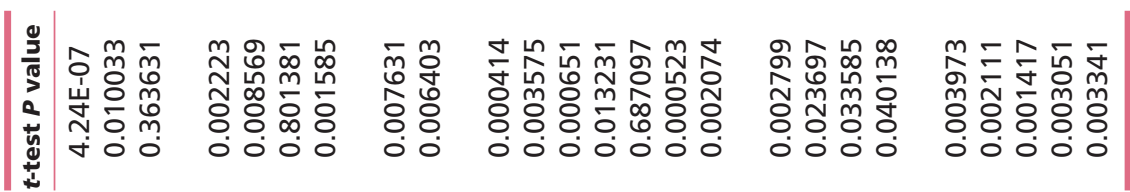

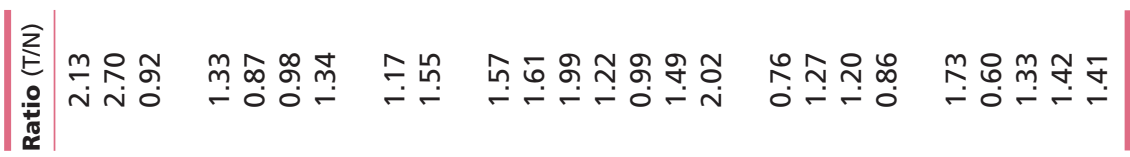

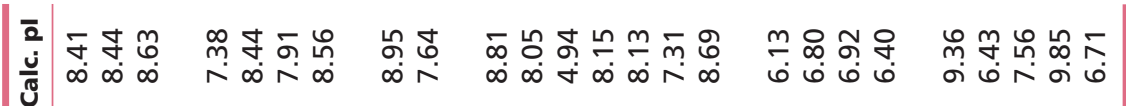

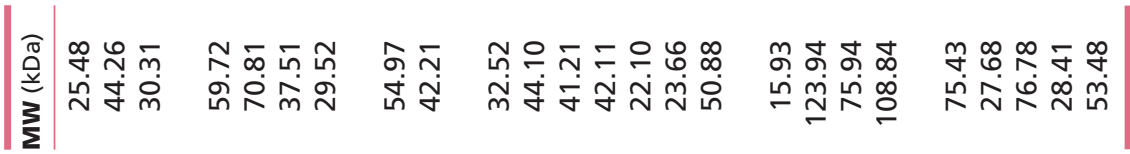

|

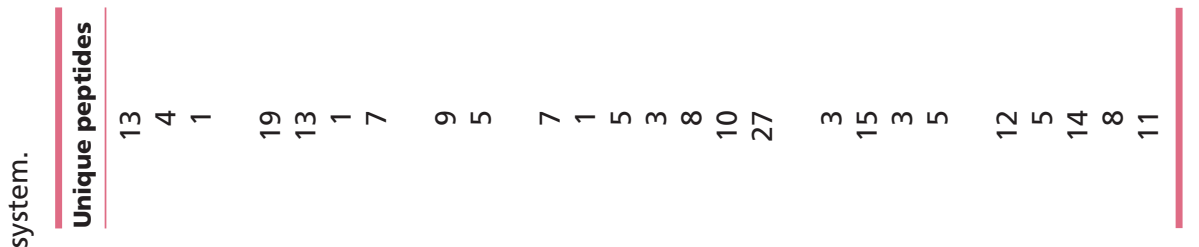

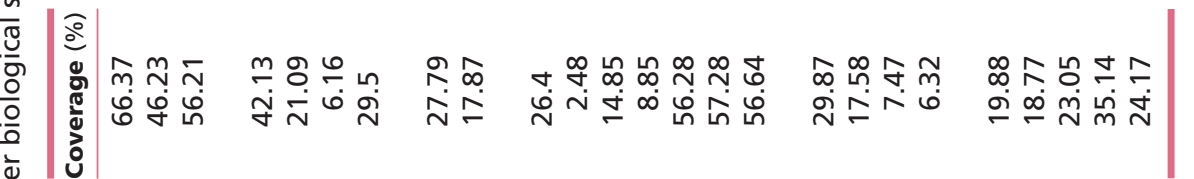

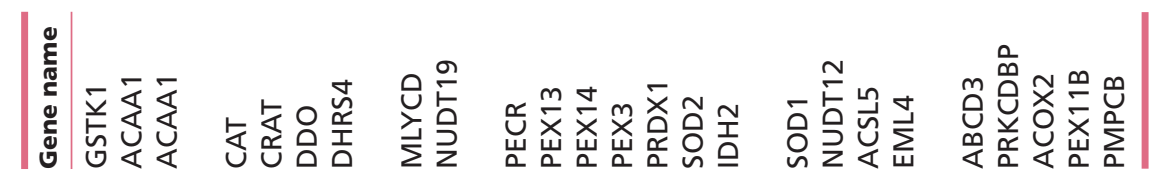

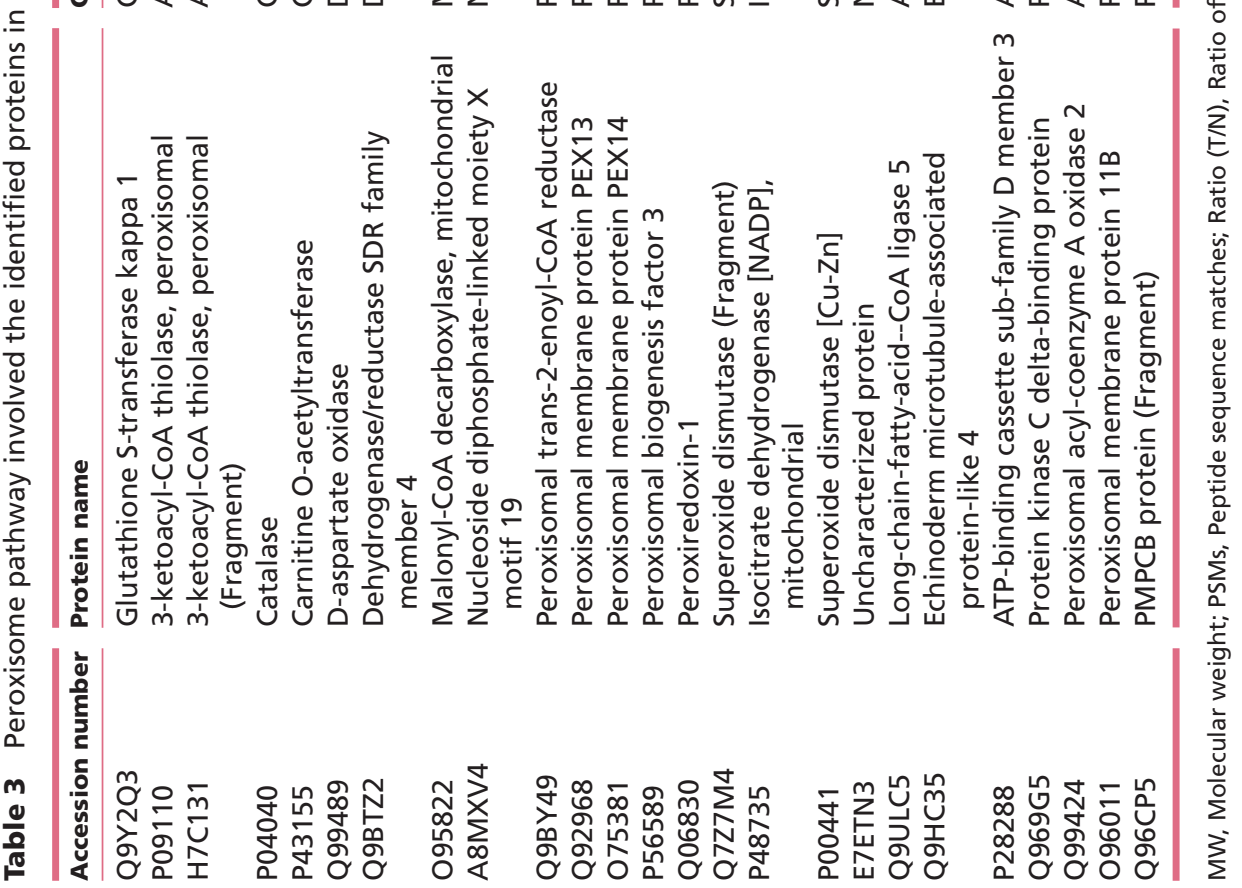




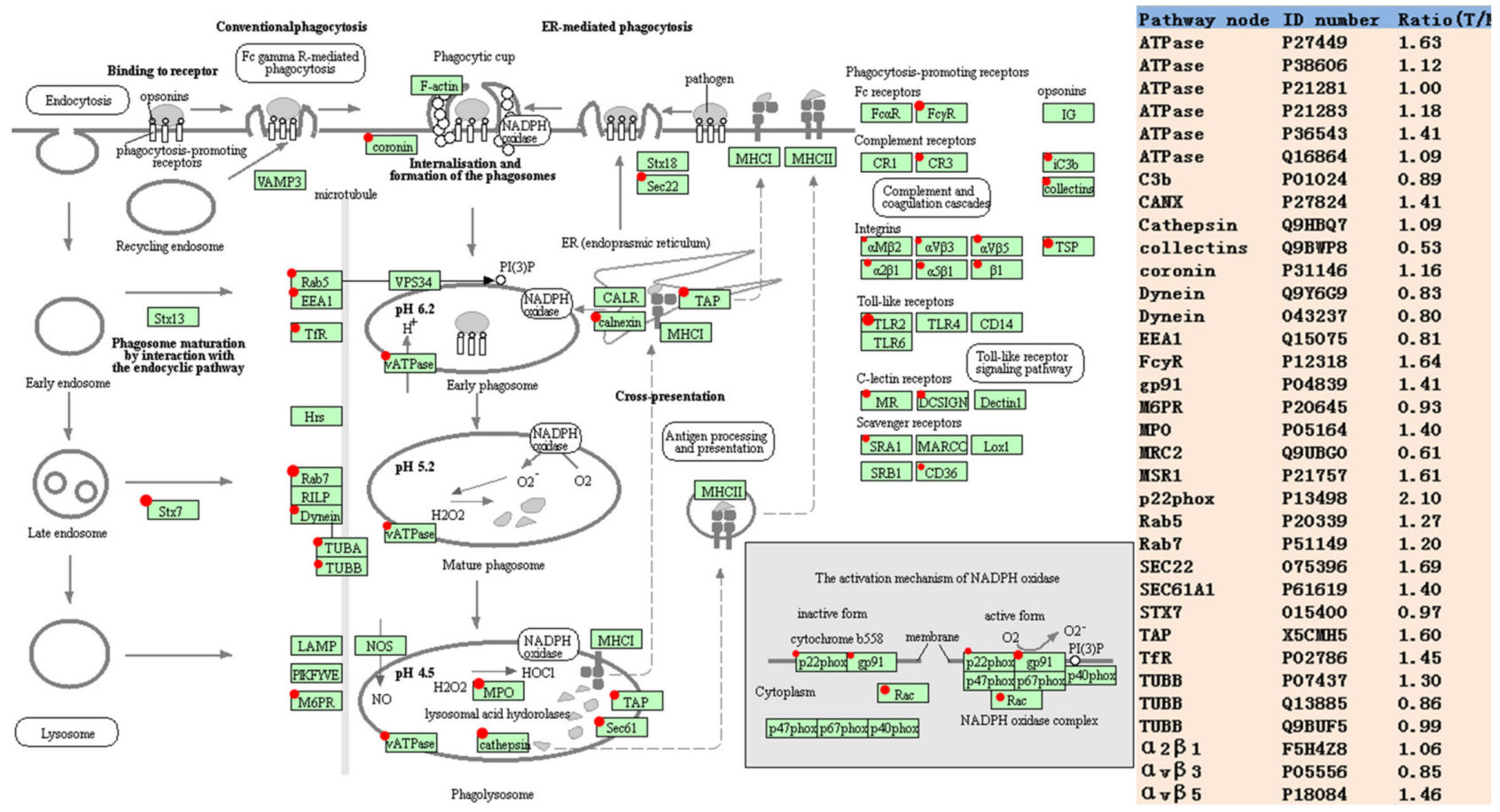

\section{Figure 4}

Phagosome pathway altered in an ovarian cancer. Green rectangle with red mark means the identified proteins. Green rectangle without red mark means species-specific enzymes. White rectangle means reference pathway. The solid line means molecular interaction. The dot line means indirect effect. The circle means mostly chemical complex. The pathway node in the right panel corresponds to the red marked node in the left diagram. ID number is the Swiss-Prot accession number. Ratio $(T / N)=$ Ratio of tumors to controls. A full colour version of this figure is available at https://doi. org/10.1530/ERC-18-0243.

invasion and metastasis (Fig. 2C). Mitophagy involves the engulfment of any material in a double-membrane enclosed autophagosome, which subsequently fuses with lysosomes. In autophagosomes, mitochondrion, proteins or peroxisome were usually observed. Autophagosomes fuse with lysosomes and emit high-energy substances, including fatty acid and amino acid (Zimmermann \& Reichert 2017). In this progress, mitophagy is dependent on the general autophagy machinery and relies on a growing cadre of 'mitophagy adaptors' and regulatory molecules, such as FUNDC1, BNIP3L(NIX), PGAM5, CK, OPA1, prohibitin2, OPTN, TBK1, p62 and Bcl2-L13 (Drake et al. 2017). This current study identified those proteins too, even though few of them appeared to have changing expression patterns (Table 2). The results were consistent with previous studies, for those proteins activated downstream mitophagy by post-translational modifications (Zimmermann \& Reichert 2017).

A peroxisome is a kind of organelle in nearly all eukaryotic cells. It is involved in metabolism of fatty acids, amino acids and polyamines, and reduction of reactive oxygen, and also contains two enzymes in the pentose phosphate pathway, so it is important for energy metabolism (Wanders \& Waterham 2006). The peroxisome-related proteins were notably increased in EOCs relative to controls (fold change >1.5), including NUDT19 (fold change $=1.54, P=0.006$ ), PECR (fold change $=1.56, P=0.0004$ ), PEX13 (fold change $=1.61$, $P=0.003$ ), ABCD3 (fold change $=1.72, P=0.003$ ), PEX14 (fold change $=1.98, P=0.0006$ ), IDH2 (fold change $=2.01$, $P=0.002$ ), GSTK1 (fold change $=2.13, P=0.0000004$ ) and ACAA1 (fold change $=2.70, P=0.01$ ) (Fig. 3 and Table 3), which indicated peroxisome was metabolically active in EOC tissues. Mitophagy is the selective degradation of mitochondria by autophagy. Defective mitochondria following stress or damage were swallowed by phagosomes (Lemasters 2005). The phagosome-related proteins were significantly increased in EOCs relative to controls (fold change $>1.5$ ), including TAP (fold change $=1.60$, $P=0.008$ ), MSR1 (fold change $=1.61, P=0.02$ ), FcyR (fold change $=1.64, P=0.005$ ), SEC22 (fold change $=1.69$, $P=0.01$ ) and p22phox (fold change $=2.10, P=0.0002$ ) (Fig. 4 and Table 4), which indicated phagosome was metabolically active in EOC tissues.

Autophagosomes fuse with lysosomes to form autolysosome, and 'goods' in autophagy has also been http://erc.endocrinology-journals.org https://doi.org/10.1530/ERC-18-0243
(C) 2018 Society for Endocrinology Published by Bioscientifica Ltd. Printed in Great Britain 


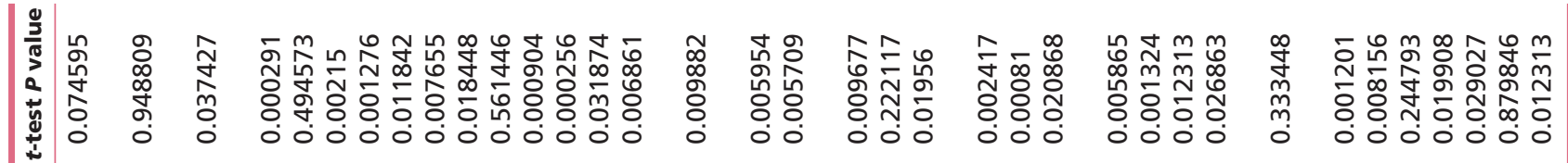

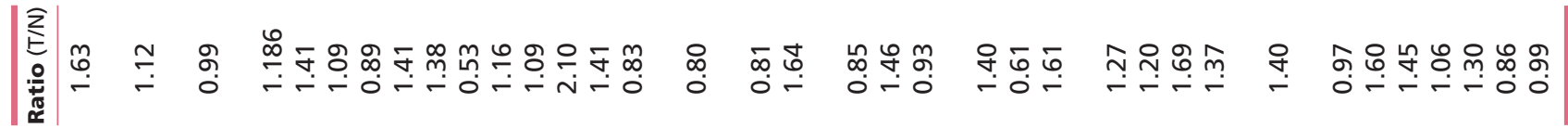

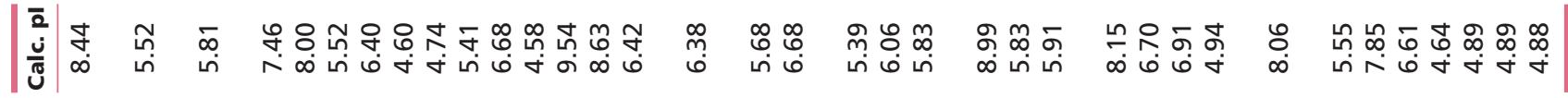

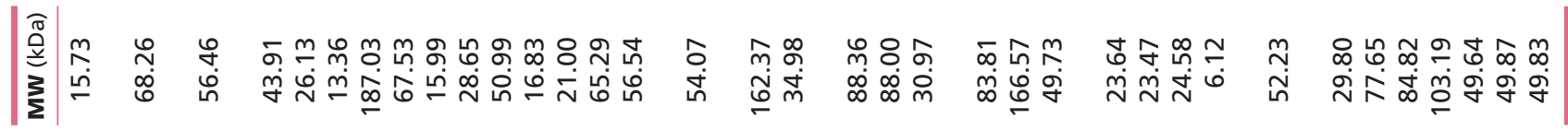

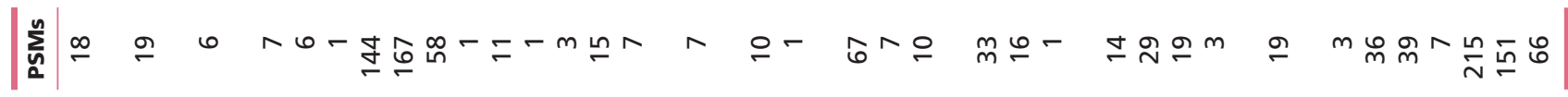

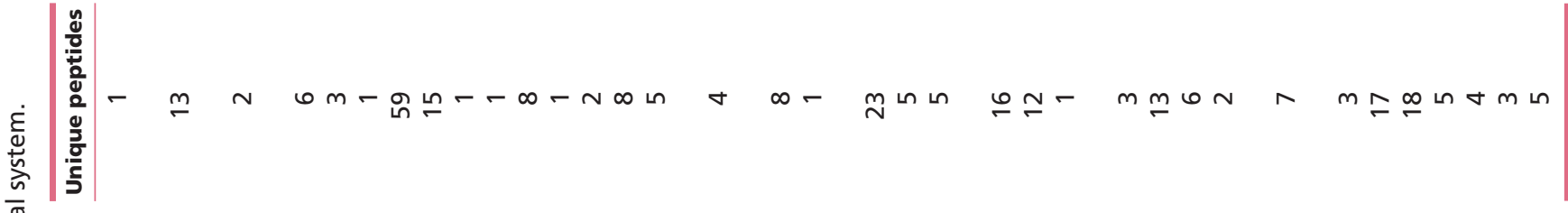

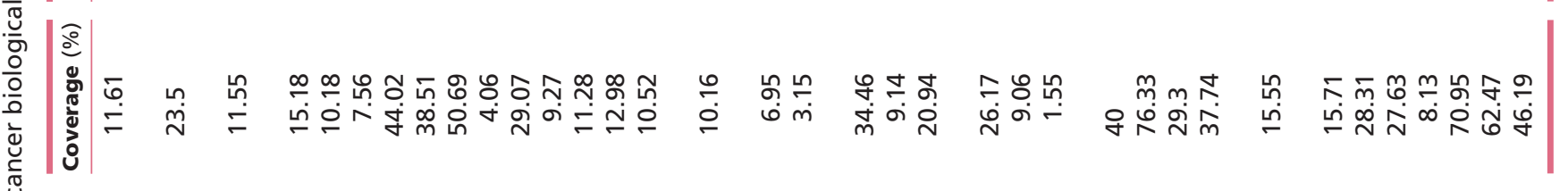

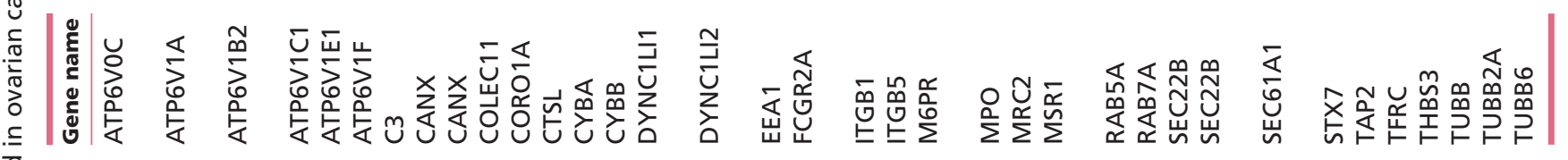

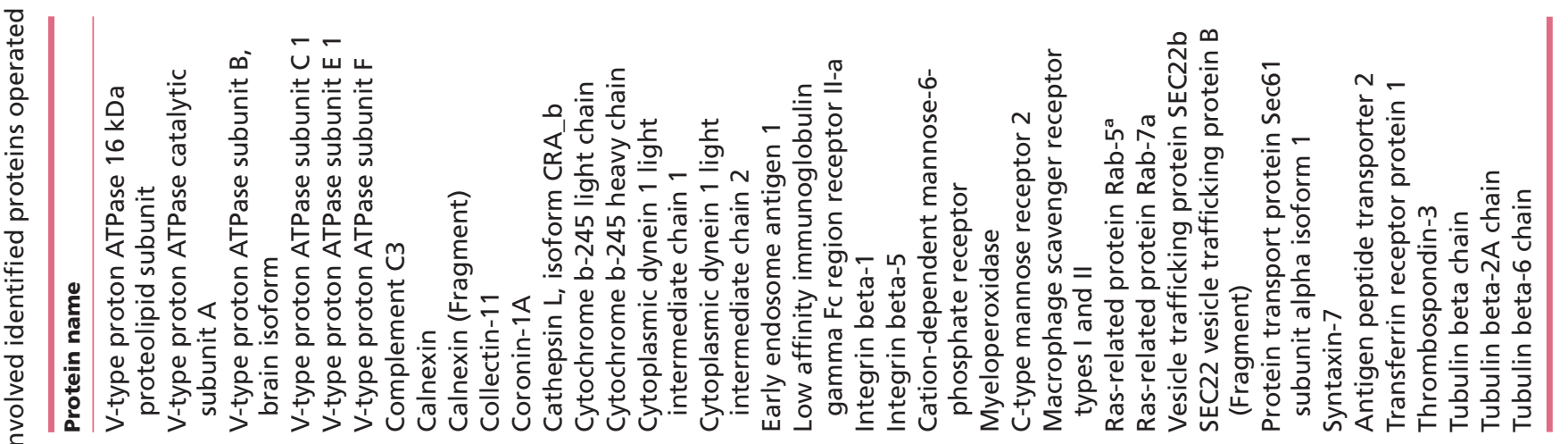

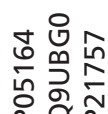

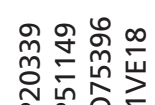

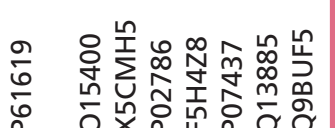



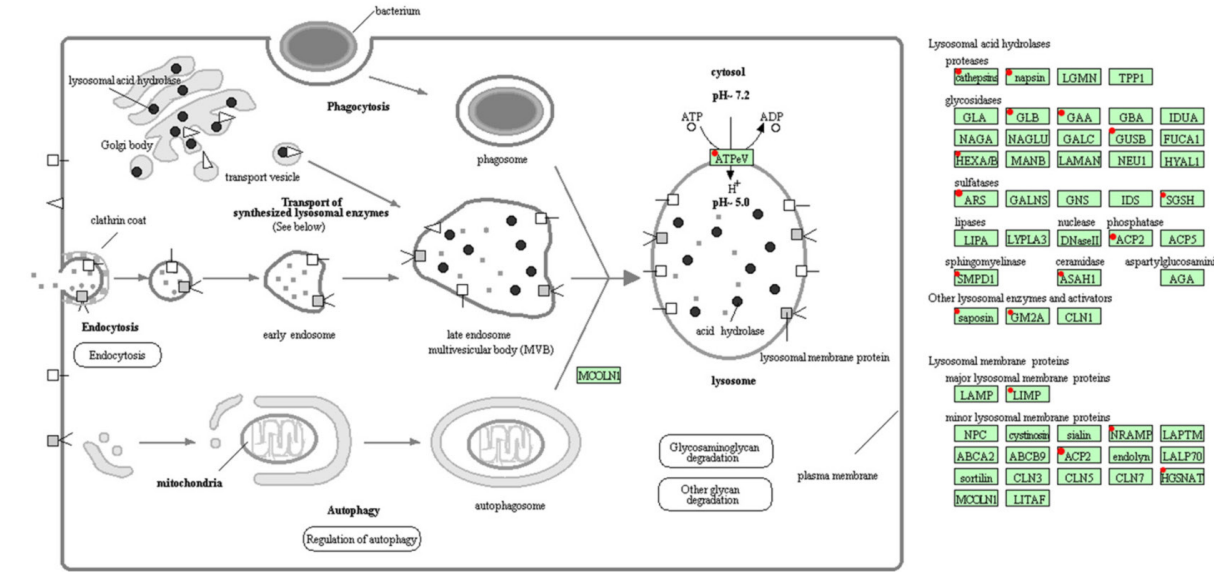

\begin{tabular}{lll}
$\begin{array}{l}\text { Pathway node } \\
\text { (protien name) }\end{array}$ & ID number & Ratio(T/N) \\
CD63 & F8VV56 & 1.76 \\
ATP6V0C & P27449 & 1.63 \\
ATP6V0A2 & Q8TBII3 & 1.40 \\
GII2A & P17900 & 1.23 \\
GIPTG & A2VDJ4 & 1.57 \\
ASAH1 & AOA1B0GTP7 & 0.77 \\
SGSH & I3NI22 & 0.68 \\
ACP2 & P11117 & 1.64 \\
AP1II2 & K7EJJ1 & 1.60 \\
AP3B2 & Q13367 & 1.28 \\
AP3D1 & Q6PK82 & 0.80 \\
FARSB & Q9BR63 & 0.70 \\
CTSD & A0A1B0GI44 & 0.78 \\
CTSG & P08311 & 1.62 \\
CLTC & Q00610 & 0.81 \\
CLTA & P09496 & 0.87 \\
GLB1 & P16278 & 1.24 \\
GAB & A0A024R8Q1 & 1.41 \\
GUSB & P08236 & 1.39 \\
HGSHAT & E5RH11 & 0.42 \\
HEXA & Q9BVJ8 & 1.42 \\
HEXB & P07686 & 1.37 \\
IGF2R & P11717 & 1.24 \\
MAPSA & IOQXC5 & 1.84 \\
PSAP & P07602 & 1.40 \\
SCARB2 & Q14108 & 1.35 \\
SLC11A2 & B3KY44 & 2.67 \\
SIIPD1 & E9LUE7 & 1.24 \\
SUIIF1 & Q8NBK3 & 1.94 \\
& & \\
\hline
\end{tabular}

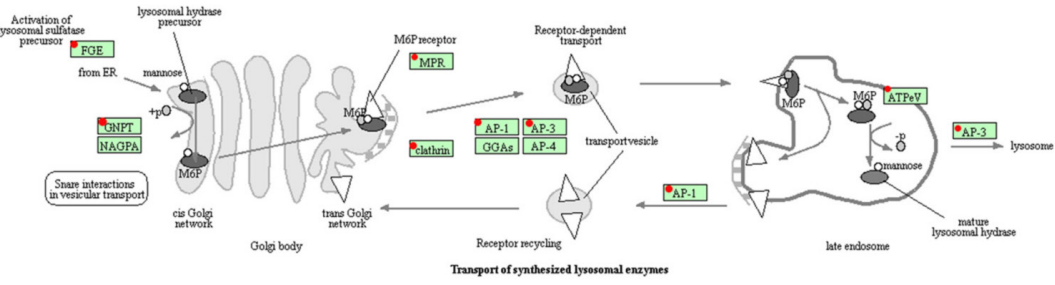

Figure 5

Lysosome pathway altered in an ovarian cancer. Green rectangle with red mark means the identified proteins. Green rectangle without red mark means species-specific enzymes. White rectangle means reference pathway. The solid line means molecular interaction. The dot line means indirect effect. The circle means mostly chemical complex. The pathway node in the right panel corresponds to the red marked node in the left diagram. ID number is the Swiss-Prot accession number. Ratio $(\mathrm{T} / \mathrm{N})=$ Ratio of tumors to controls. A full colour version of this figure is available at https://doi.org/10.1530/ERC-180243.

degraded into products (fatty acids and amino acids). Those kind of high-energy substances were transported to the cytoplasm of cells for reuse (Lemasters 2005). The lysosome pathway-related proteins were significantly increased in EOCs relative to controls (fold change $>1.5$ ), including CD63 (fold change $=1.76, P=0.007948$ ), ATP6VOC (fold change $=1.63, P=0.000424$ ), GNPTG (fold change $=1.57, P=0.018055$ ) and ACP2 (fold change $=1.64$, $P=0.006068$ ), AP1M2 (fold change $=1.603138$, $P=0.003299$ ), CTSG AP1M2 (fold change $=1.62$, $P=0.029695$ ) (Fig. 5 and Table 5), which indicated lysosome pathway was metabolically active in EOC tissues. The fatty acid degradation-related proteins were significantly increased in EOCs relative to controls (fold change $>1.5$ ), including ECHS1 (fold change $=1.52, P=0.003$ ), EHHADH (fold change $=1.62, P=0.002$ ), ECI1 (fold change $=1.64$, $P=0.001$ ) and CPT2 (fold change $=2.05, P=0.019$ ) (Fig. 6 and Table 6), which indicated fatty acid degradation pathway was metabolically active in EOC tissues.

The valine, leucine and isoleucine degradationrelated proteins were significantly increased in EOCs relative to controls (fold change $>1.5$ ), including ECHS1 (fold change $=1.52, P=0.003$ ), $\mathrm{HIBCH}$ (fold change $=1.58$, $P=0.002$ ), EHHADH (fold change $=1.61, P=0.002$ ),
IL4I1 (fold change=1.82, $P=0.008$ ), HMGCS2 (fold change $=2.17, P=0.002$ ) and ACAA1 (fold change $=2.70$, $P=0.0100$ ) (Fig. 7 and Table 7), which indicated valine, leucine and isoleucine degradation pathway was metabolically active in EOC tissues.

\section{qRT-PCR and Western blotting validated the consistency of iTRAQ quantitative mitochondrial proteomics}

To validate the DEPs identified by iTRAQ quantitative mitochondrial proteomics, we examined the protein expressions of the identified DEPs, including GLDC, PCK2, IDH2, CPT2 and HMGCS2 in the mitochondrial protein samples prepared from EOC and control tissues, and the mRNA and protein expressions of those five DEPs in the cultured EOC cells TOV21G and control cells IOSE80. Except for HMGCS2, a significant increase in the mRNA and protein expression levels of GLDC, PCK2, IDH2 and CPT2 was observed in the cultured cells by q-PCR and Western blot, respectively (Fig. 8A and B). The results of Western blot in the prepared mitochondrial samples had a good consistency with the results of iTRAQ quantitative mitochondrial proteomics (Fig. 8C). 


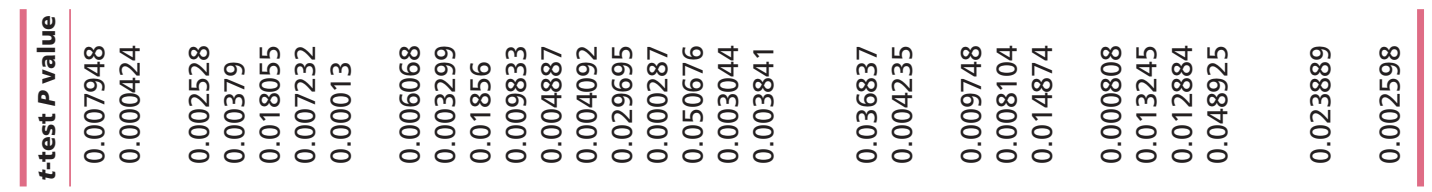

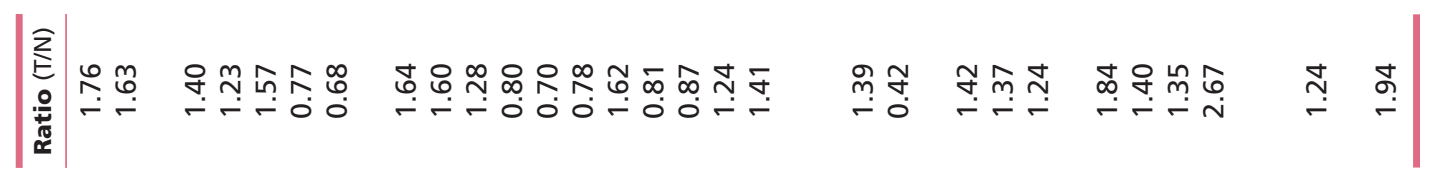

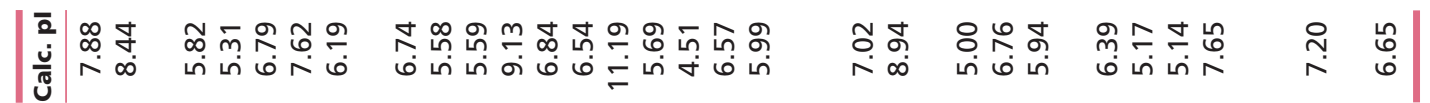

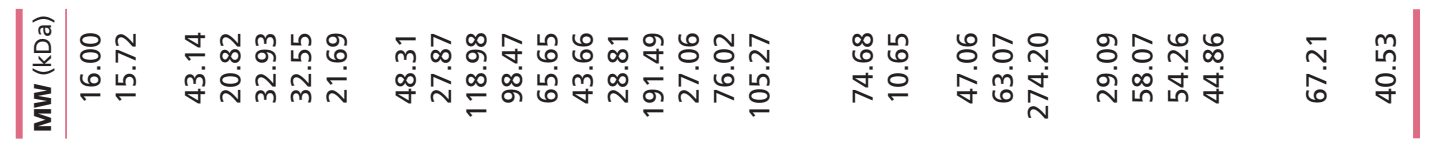

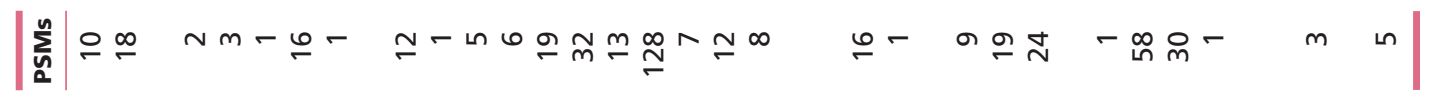

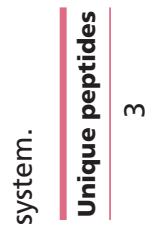

$a-\operatorname{Lㅇㅛ~}$

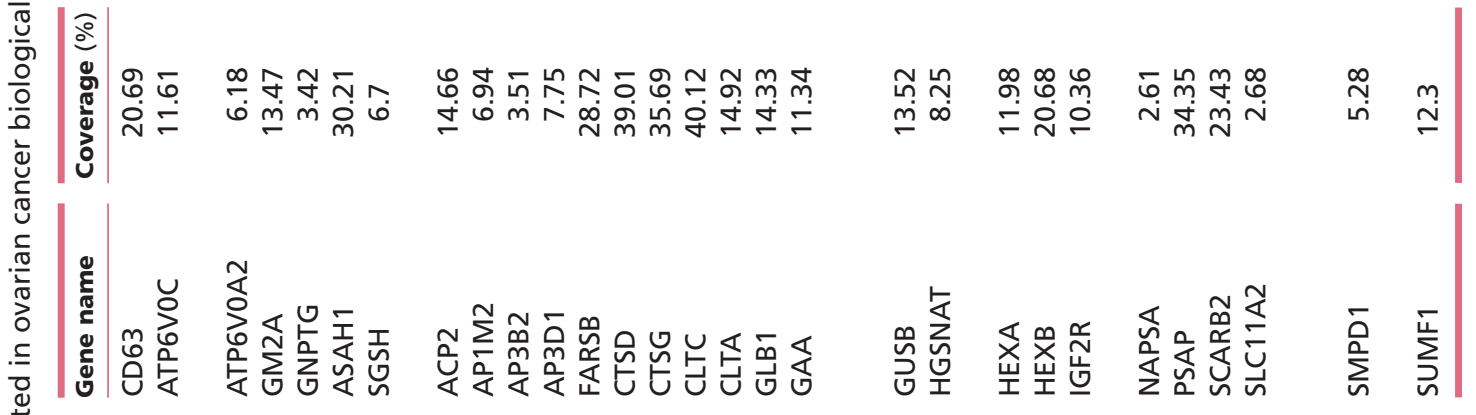

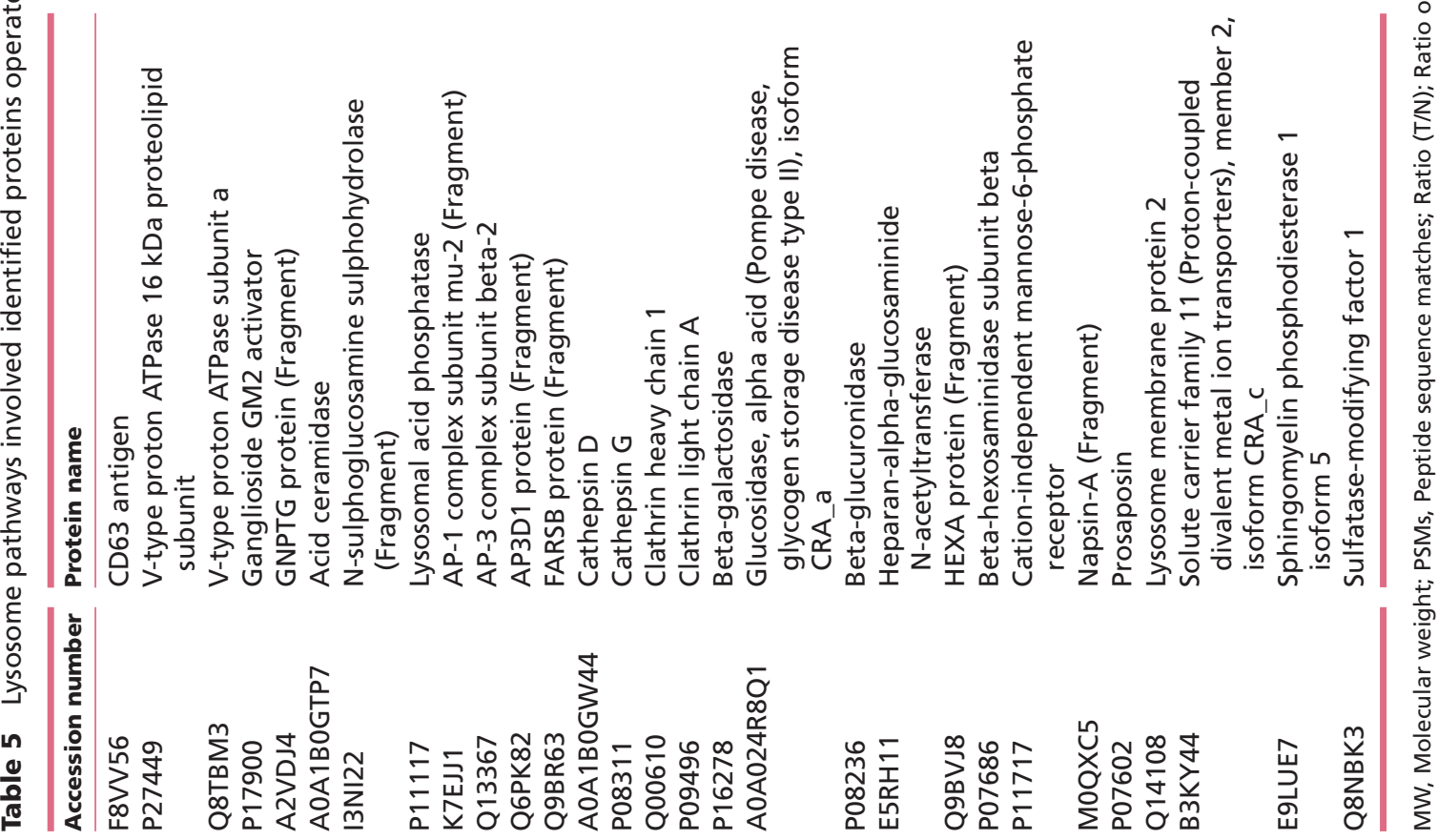



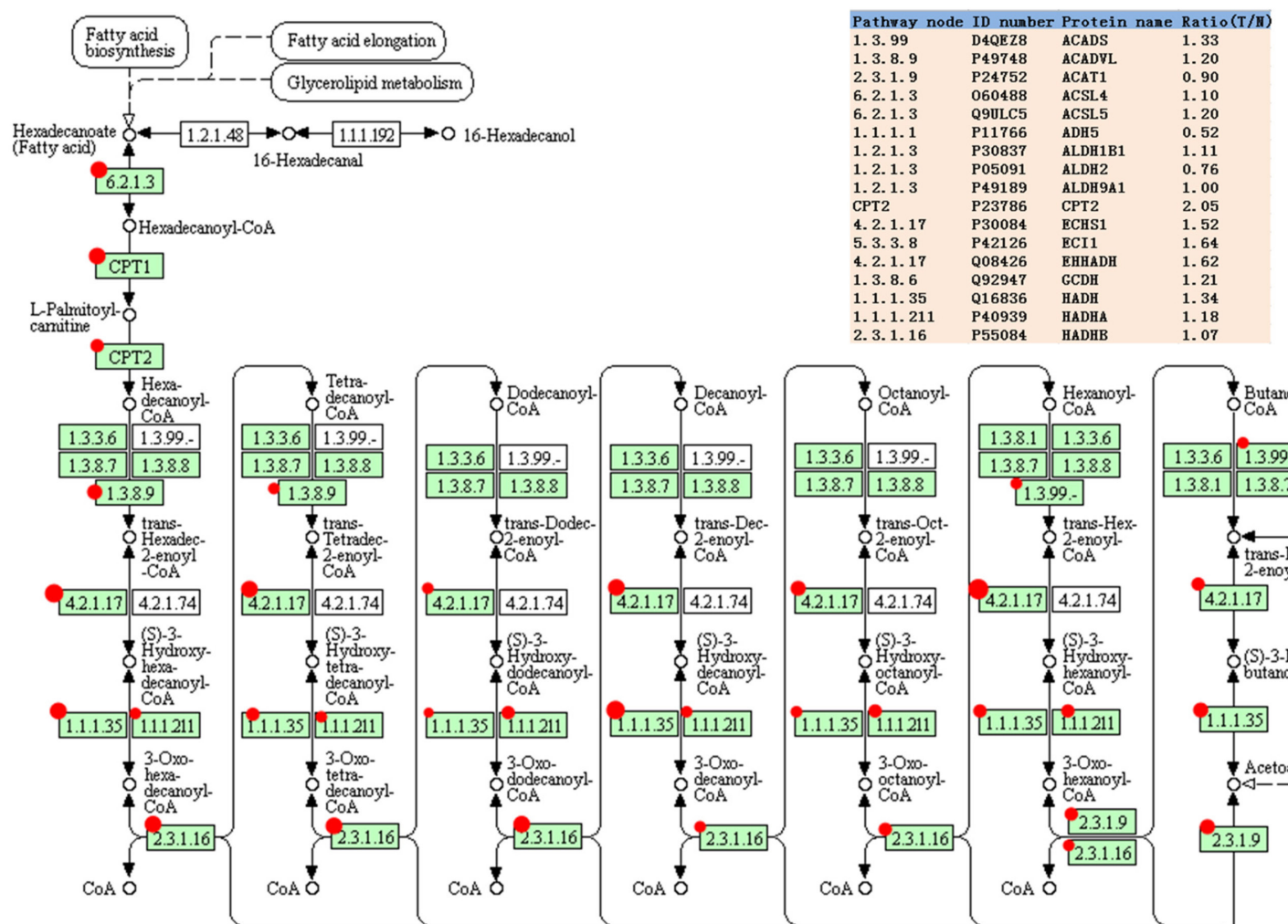

$\begin{array}{lll} & \\ & & \\ \end{array}$
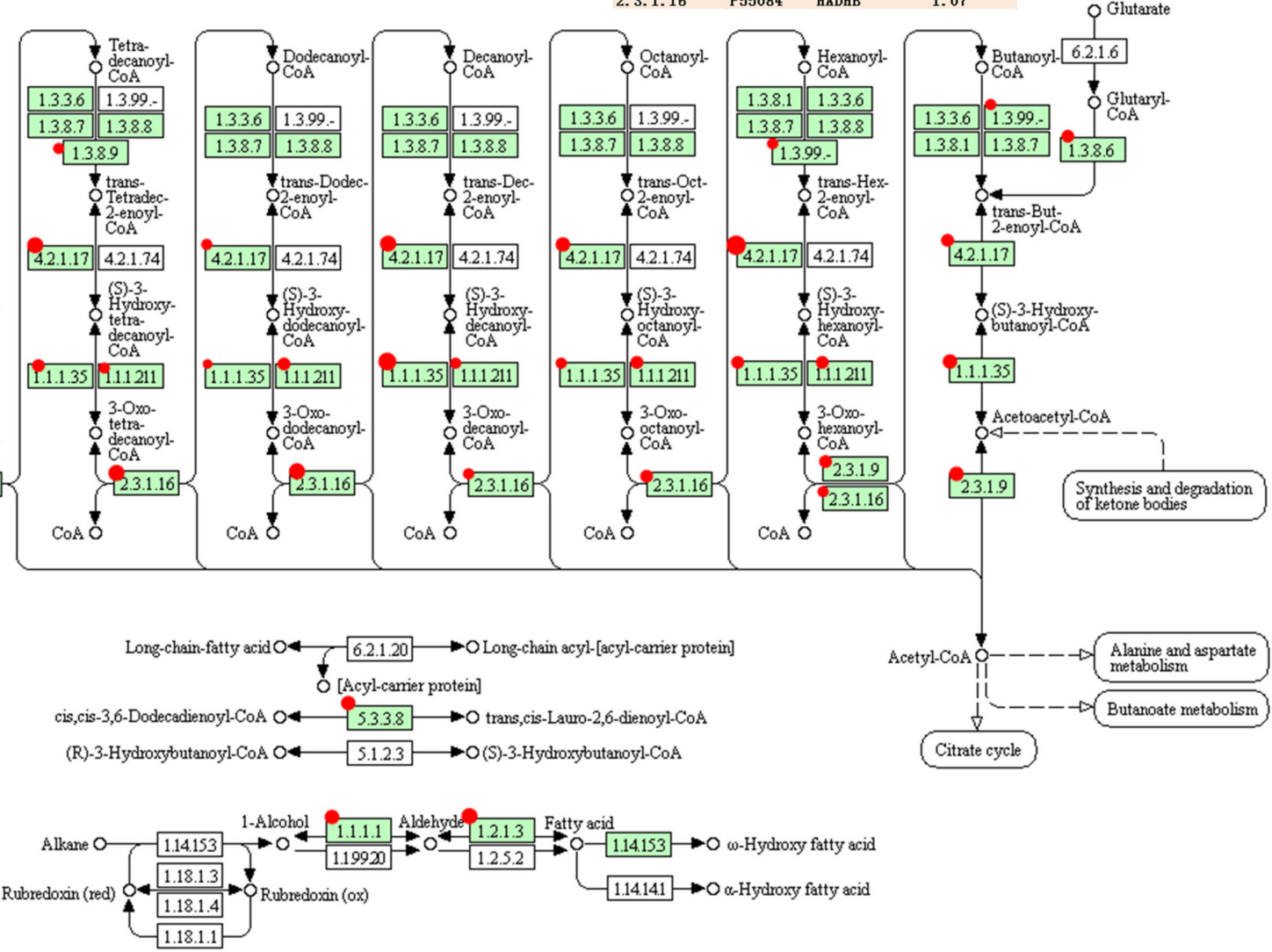

\section{Figure 6}

Fatty acid degradation pathway altered in an ovarian cancer. Green rectangle with red mark means the identified proteins. Green rectangle without red mark means species-specific enzymes. White rectangle means reference pathway. The solid line means molecular interaction. The dot line means indirect effect. The circle means mostly chemical complex. The pathway node in the upper panel corresponds to the red marked node in the lower diagram. ID number is the Swiss-Prot accession number. Ratio $(T / N)=$ Ratio of tumors to controls. A full colour version of this figure is available at https://doi. org/10.1530/ERC-18-0243.

\section{Discussion}

EOC is the leading cause of death from gynecologic cancer and the concealed characteristics caused difficulty to be diagnosed in the early stage (Chan et al. 2006). The symptoms index plus CA125 screening may be the best way to identify women who may have an EOC (Karabudak et al. 2013). However, the 5-year overall survival rate for patients diagnosed with stage III-IV EOC remains still poor (about 30\%) (Miller et al. 2016). Joint detection of different tumor biomarkers is necessary to increase the specificity of the immunoassay. Proteomics provides a feasible approach for large-scale screening of EOC-related proteins to enhance the understanding of EOC pathogenesis. There are few reports on proteomics analysis of EOCs (Hiramatsu et al. 2016). Until now, it is the first time to use iTRAQ-based quantitative proteomics to identify EOC-related proteins in mitochondria. Mitochondria play a central role in the regulation of cellular signals, metabolism and apoptosis in cancer cells. In this study, one aimed at identifying the potential mitochondrial biomarkers for the prediction, prevention, 


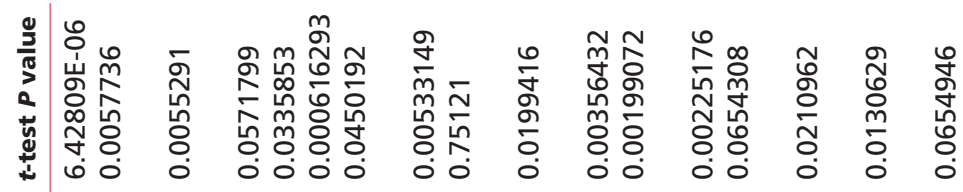

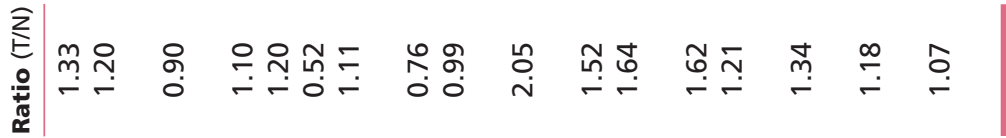

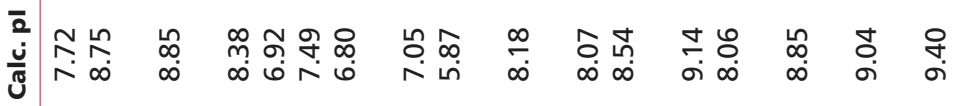

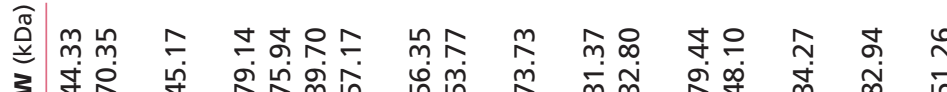

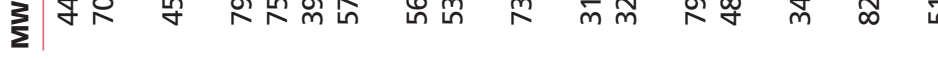

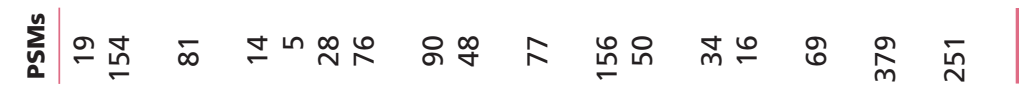

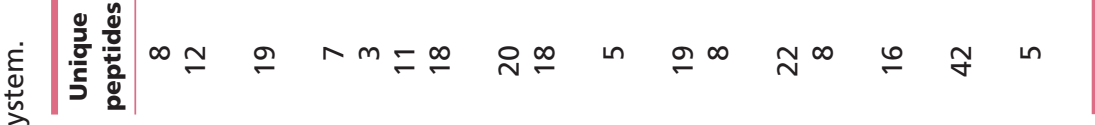

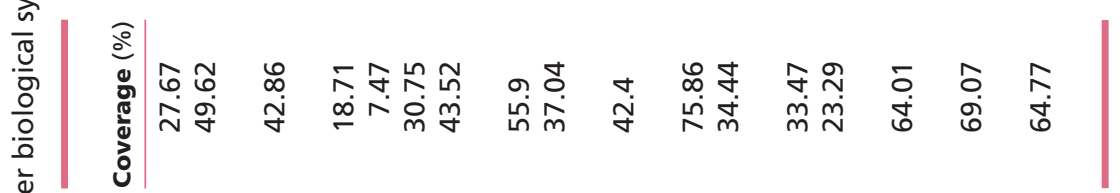

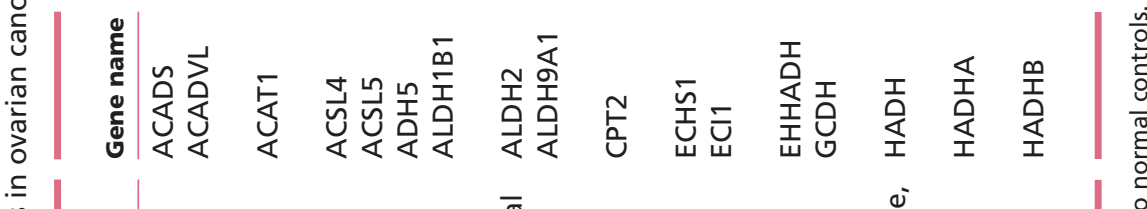

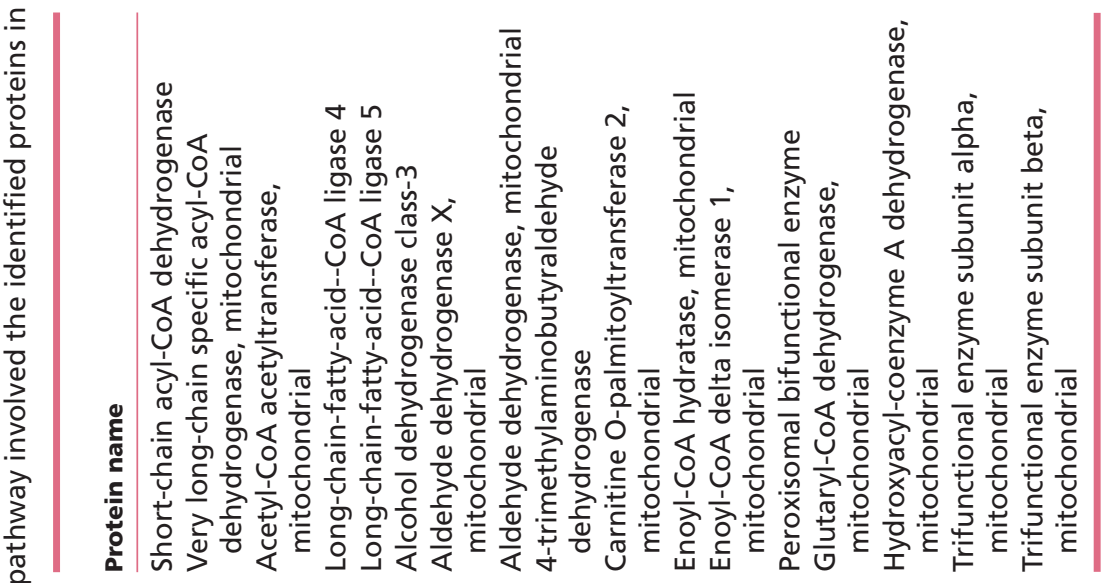

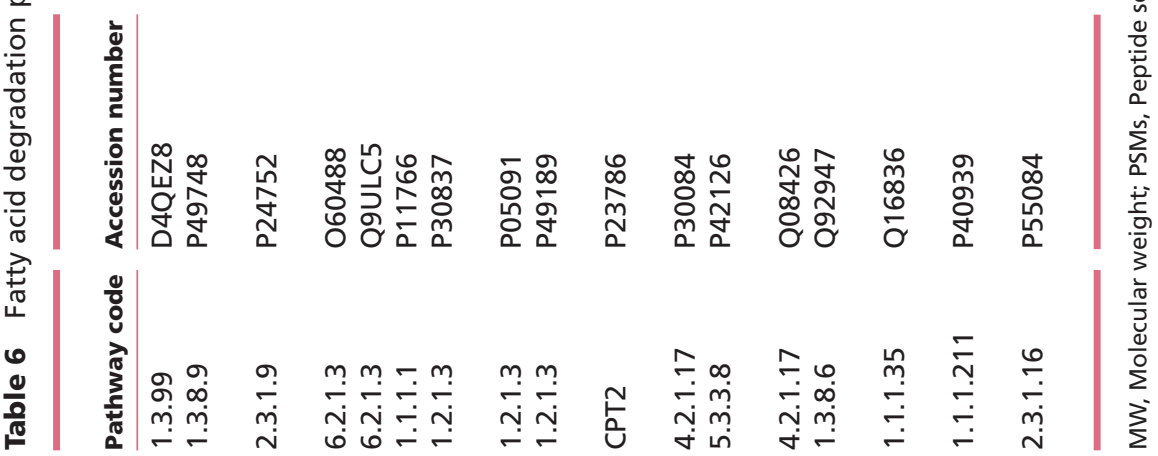




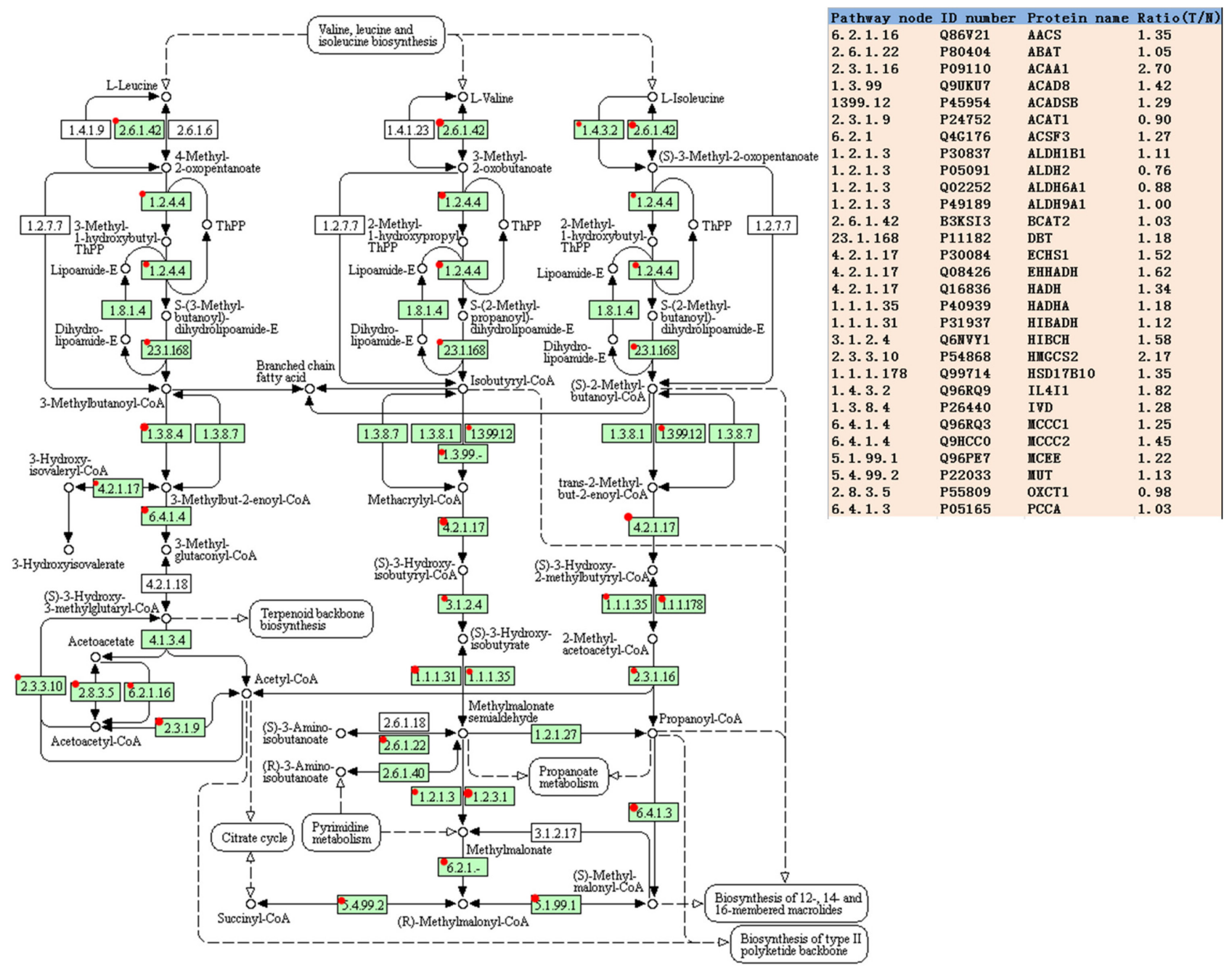

Figure 7

Valine, leucine and isoleucine degradation pathway altered in an ovarian cancer. Green rectangle with red mark means the identified proteins. Green rectangle without red mark means species-specific enzymes. White rectangle means reference pathway. The solid line means molecular interaction. The dot line means indirect effect. The circle means mostly chemical complex. The pathway node in the right panel corresponds to the red marked node in the left diagram. ID number is the Swiss-Prot accession number. Ratio $(\mathrm{T} / \mathrm{N})=$ Ratio of tumors to controls. A full colour version of this figure is available at https://doi.org/10.1530/ERC-18-0243.

diagnosis and treatment of EOCs. Mitochondrial purification was progressed by Nycodenz density gradient approach, followed by an 6-plex iTRAQ proteomics to identify mtEPs in EOCs relative to controls. Among 5115 iTRAQ-identified proteins, 262 proteins were significantly related to overall survival in EOC patients. The iTRAQ and TCGA data were integrated, and GO analysis, proteinprotein interaction and gene coexpression were analyzed. Moreover, 63 proteins were identified as potential markers for the development of an EOC, such that ERBB2, PTBP1 and H2AFX as reported were significantly related to overall survival. To some degree, these results indicate that our findings were consistent with previous studies and also make new discoveries. Moreover, we searched
CooLGeN database with key words of 'marker or biomarker' and 'cancer' to understand the situation of our findings in the research of other cancers, not only EOCs. One found that 17 biomarkers had been reported in other cancers, including ANXA2, TACSTD2, TNFSF10, HNRNPA2B1, RBP4, PPP1R13L, UGT8, VTN, BCAT1, PTPN2, PDIA6, MFAP4, GSTZ1, NUCB2, P4HB and PTBP1, which indicated our new found biomarkers were reliable. Numerous reported EOC protein biomarkers such as MUC16, MSLN, ERBB2, CHI3L1, MUC1, CD44, VTCN1 and CRP (Simon et al. 2007, Chiang et al. 2015, Stewart et al. 2015, Jiang et al. 2017, Bartakova et al. 2018, Fortner et al. 2018) were also identified in this iTRAQ proteomics study. It demonstrated that iTRAQ proteomic strategy is http://erc.endocrinology-journals.org https://doi.org/10.1530/ERC-18-0243
C) 2018 Society for Endocrinology Published by Bioscientifica Ltd. Printed in Great Britain 


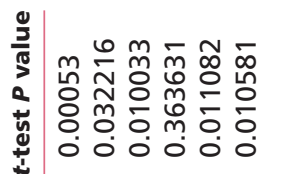

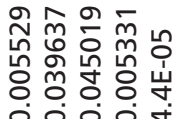

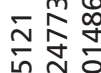

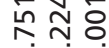

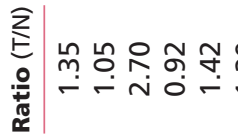

ำำ

ㅇํㅇ음

高

ब.

缉比守侘夺字

in

을

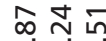

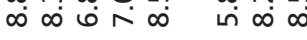

ध

ㅇำ

ผึ่

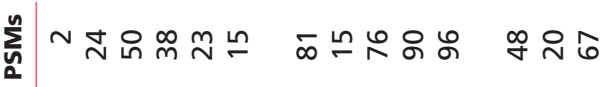

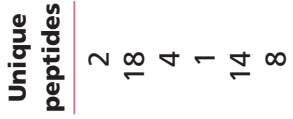

도용ํำ

$\stackrel{\infty}{\leftarrow} \stackrel{\infty}{\sim}$

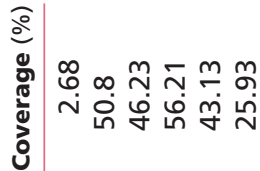

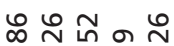

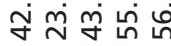

$\dot{\infty} \stackrel{\infty}{\wedge} \stackrel{\infty}{m}$ $\hat{m} \dot{m} \stackrel{\infty}{m}$

œ于 千 ก่ำ

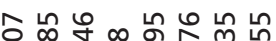

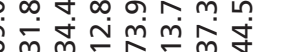

จิ โิ $\stackrel{n}{\sim} \simeq$

กิ

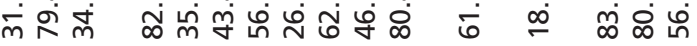

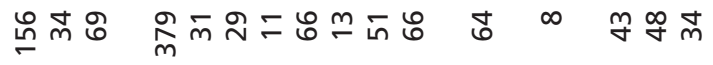

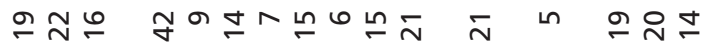

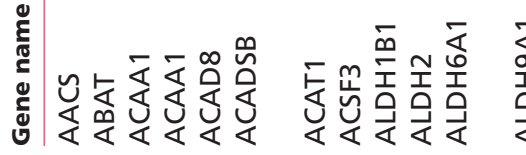
交 甹豆至

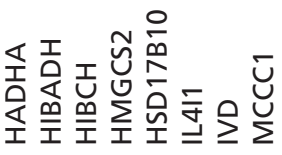

$\overline{\frac{\pi}{2}} \cdot \frac{\bar{\pi}}{\frac{2}{2}} \cdot \frac{\overline{\frac{\pi}{2}}}{\frac{2}{2}}$

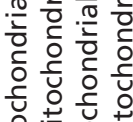

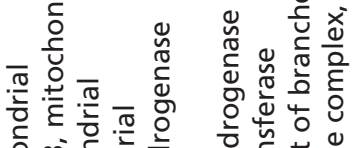

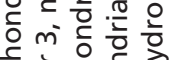

过

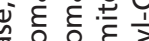

政

है. 등 d

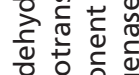

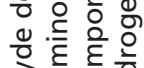

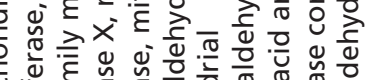

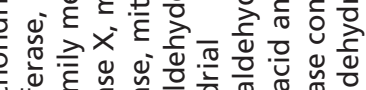

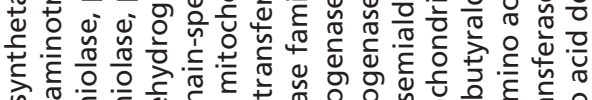

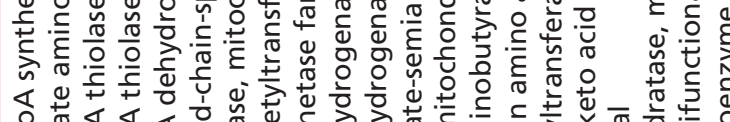

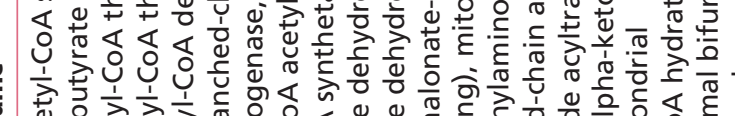

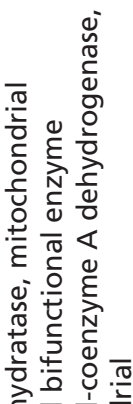
. बं है

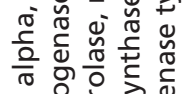

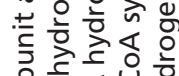

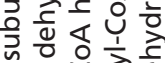

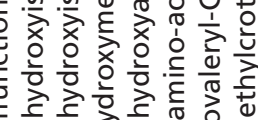
+ $m m \dot{m} m$

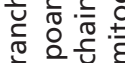

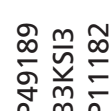

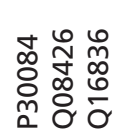

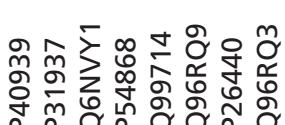

원

m̌n

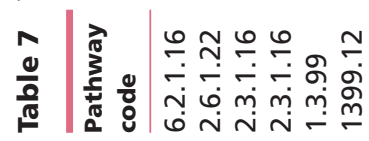

mุi

$m \stackrel{\infty}{*}$

ำำ

īi

ṃn

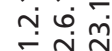


A

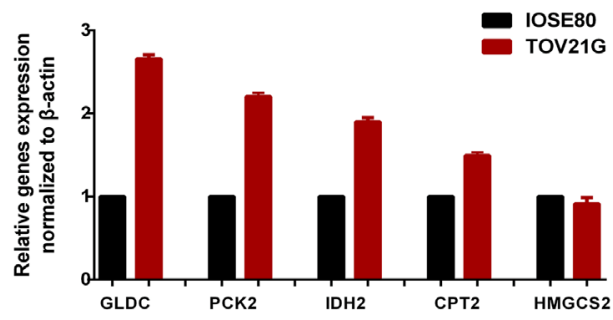

B

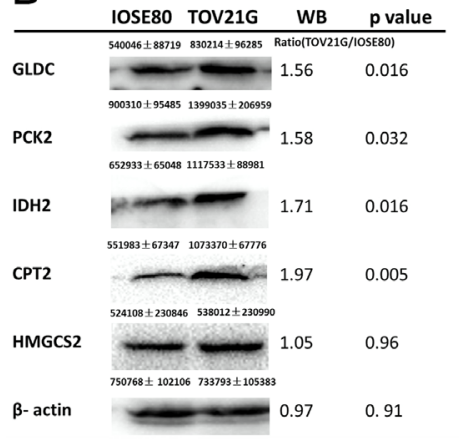

C

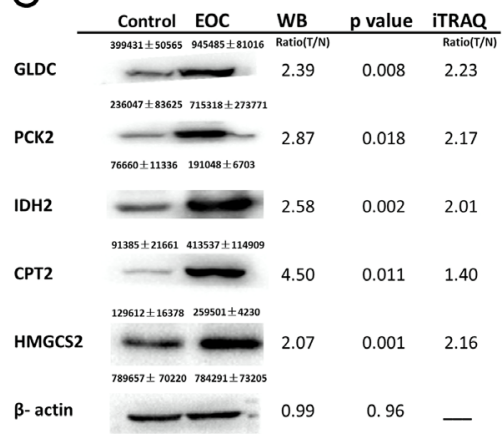

Figure 8

qRT-PCR and Western blot analyses to validate results of iTRAQ quantitative mitochondrial proteomics. (A) qTR-PCR analysis to quantify the expression levels of GLDC, PCK2, IDH2, CPT2 and HMGCS2 between EOC cells TOV21G and control cells IOSE80. (B) Protein expression levels of GLDC, PCK2, IDH2, CPT2 and HMGCS2 in EOC cells TOV21G and control cells IOSE80. (C) Mitochondrial proteins of EOC and control tissues were analyzed by Western blot using antibodies against GLDC, PCK2, IDH2, CPT2 and HMGCS2. The levels of GLDC, PCK2, IDH2, CPT2 and HMGCS2 were normalized relative to $\beta$-actin. Data represent mean \pm s.D. A full colour version of this figure is available at https://doi.org/10.1530/ERC-18-0243.

a reliable tool for identity of EOC biomarker. Compared to previous studies, 992 proteins of 5115 identified proteins have been reported to associate ovary checked by CooLGeN database. Among 992 proteins, there were 33 proteins upregulated more than two-fold; and 12 of those 33 identified proteins have not been reported in previous EOC biomarker studies, including IDH2, RAB43, SYNGR2, RDH10, CYBA, HMGCS2, PCK2, RIDA, KIF23, UGT8, SC11A2 and AIG1, which indicates that those proteins need further studies to testify them as novel EOC biomarkers. In the documented study, most of them endeavor on the effect of single factor or gene mutation on the development of cancer. However, some studies found that only one single molecular event does not lead to the occurrence of cancer. A typical cancer occurrence model needs the mutation of two to eight driver genes (van der Wal et al. 2018). In this study, a recognized biomarker pattern should be put forward, and it means that the use of a set of biomarkers builds a data model to improve the accuracy and specificity of prediction, diagnosis, prognosis and therapy of an EOC. Despite its common use in cancer treatment, biomarkers have not really entered the era of precision medicine to predict, prevent and personalize the treatment of an EOC, and there have been no approaches to adjust personalized medicine based on biological differences between or within tumors. A genome-based model for adjusting radiotherapy dose (GARD), which was reported in 2017, has attracted much attention (Scott et al. 2017). One is going to use the gene expression-based drug-reaction index and the linear quadratic model to derive the drug dose, which one speculates would relate to clinical outcome. In the future, it is necessary for one to collect prospective clinical data to validate the model.

Mitophagy involves the engulfment of any material in a double-membrane enclosed autophagosome, which subsequently fuses with lysosomes. In autophagosomes, mitochondria, proteins or peroxisomes were usually observed. Autophagosomes fuse with lysosomes and emit high-energy substances, including fatty acids and amino acids (Zimmermann \& Reichert 2017). Pathway network analysis mapped the identified proteins to 70 signaling pathways. Interestingly, the results demonstrated that cancer cells exhibited an increased dependence on mitophagy, such as peroxisome, phagosome, valine, leucine and isoleucine degradation, fatty acid degradation pathway, which might play an important role in EOC invasion and metastasis. In this progress, mitophagy is dependent on the general autophagy machinery and relies on a growing cadre of 'mitophagy adaptors' and regulatory molecules, such as FUNDC1, BNIP3L(NIX), PGAM5, CK, OPA1, prohibitin 2, OPTN, TBK1, p62 and Bcl2-L13 (Drake et al. 2017). This study identified those proteins too, even though few of them appeared expression changing. The results were consistent with previous studies, because those proteins activated downstream mitophagy by post-translational modifications (Wu et al. 2016). Two mitophagy mechanisms in mammalian cells had been reported. Firstly, receptor-mediated mitophagy is activated by phosphorylation, increasing its binding proteins for Atg8-like and recruiting them to mitochondria. Secondly, the highly ubiquitylated mitochondrial outer membrane proteins recruit adapter proteins, which in turn recruits Atg8-like proteins. For NIX (BNIP3L), BNIP3 and 
BCL2L13, only the activated phosphorylation mechanism and the modified residues are known but the kinases and phosphatases have not been identified; yet, for FUNDC1, both the activated and deactivated phosphorylation mechanisms, the modified residues and participated enzymes are known (Zimmermann \& Reichert 2017). In this regard, one could not ignore those identified proteins without significant change. In the future, more and more proteins experienced post-translational modifications might also be biomarker, even become more effective (Peng et al. 2015).

In conclusion, this study used iTRAQ-SCX-LC-MS/MS to identify and quantify $5115 \mathrm{mtEPs}$ in EOCs relative to controls. The first quantitative reference map of a human EOC tissue mitochondrial proteome has been achieved here. These findings enriched human mitochondrial database and a series of identified biomarkers could be useful for clinical practice. These results demonstrated that iTRAQ-SCX-LC-MS/MS is a reliable method to identify and quantify mitochondrial protein profiles and could assist in exploiting potential biomarkers and the novel mechanisms of EOC carcinogenesis.

\section{Supplementary data}

This is linked to the online version of the paper at https://doi.org/10.1530/ ERC-18-0243.

\section{Declaration of interest}

The authors declare that there is no conflict of interest that could be perceived as prejudicing the impartiality of the research reported.

\section{Funding}

This work was supported by the Xiangya Hospital Funds for Talent Introduction (to $X \mathrm{Z}$ ), the Hunan Provincial Hundred Talent Plan (to X Z), the grants from China '863' Plan Project (Grant No. 2014AA020610-1 to X Z), the National Natural Science Foundation of China (Grant No. 81272798 and 81572278 to $X Z$ ) and the Hunan Provincial Natural Science Foundation of China (Grant No. 14JJ7008 to X Z).

\section{Author contribution statement}

$\mathrm{N} \mathrm{L}$ analyzed data, prepared figures and tables, designed and wrote the manuscript. $\mathrm{H} \mathrm{L}$ collected samples and prepared mitochondrial samples. $\mathrm{L} C$ collected tumor tissue samples and performed clinical diagnosis. $\mathrm{X} Z \mathrm{Z}$ conceived the concept, designed experiments and manuscript, instructed experiments and data analysis, supervised results, coordinated, critically revised/wrote manuscript and was responsible for its financial supports and the corresponding works. All authors approved the final manuscript.

\section{References}

Bartakova A, Michalova K, Presl J, Vlasak P, Kostun J \& Bouda J 2018 CD44 as a cancer stem cell marker and its prognostic value in patients with ovarian carcinoma. Journal of Obstetrics and Gynaecology 38 110-114. (https://doi.org/10.1080/01443615.2017.1336753)

Borgfeldt C, Bendahl PO, Ferno M \& Casslen B 2003 High preoperative plasma concentration of tissue plasminogen activator (tPA) is an independent marker for shorter overall survival in patients with ovarian cancer. Gynecologic Oncology 91 112-117. (https://doi. org/10.1016/S0090-8258(03)00493-1)

Chan JK, Cheung MK, Husain A, Teng NN, West D, Whittemore AS, Berek JS \& Osann K 2006 Patterns and progress in ovarian cancer over 14 years. Obstetrics and Gynecology 108 521-528. (https://doi. org/10.1097/01.AOG.0000231680.58221.a7)

Chen W, Zheng R, Baade PD, Zhang S, Zeng H, Bray F, Jemal A, Yu XQ \& He J 2016 Cancer statistics in China, 2015. CA: A Cancer Journal for Clinicians 66 115-132. (https://doi.org/10.3322/caac.21338)

Cheng T \& Zhan X 2017 Pattern recognition for predictive, preventive, and personalized medicine in cancer. EPMA Journal 8 51-60. (https:// doi.org/10.1007/s13167-017-0083-9)

Chiang YC, Lin HW, Chang CF, Chang MC, Fu CF, Chen TC, Hsieh SF, Chen CA \& Cheng WF 2015 Overexpression of CHI3L1 is associated with chemoresistance and poor outcome of epithelial ovarian carcinoma. Oncotarget 6 39740-39755. (https://doi.org/10.18632/ oncotarget.5469)

Cleland TP 2018 Solid digestion of demineralized bone as a method to access potentially insoluble proteins and post-translational modifications. Journal of Proteome Research 17 536-542. (https://doi. org/10.1021/acs.jproteome.7b00670)

Cymbaluk-Ploska A, Chudecka-Glaz A, Pius-Sadowska E, Machalinski B, Menkiszak J \& Sompolska-Rzechula A 2018 Suitability assessment of baseline concentration of MMP3, TIMP3, HE4 and CA125 in the serum of patients with ovarian cancer. Journal of Ovarian Research 11 1. (https://doi.org/10.1186/s13048-017-0373-9)

Drake LE, Springer MZ, Poole LP, Kim CJ \& Macleod KF 2017 Expanding perspectives on the significance of mitophagy in cancer. Seminars in Cancer Biology 47 110-124. (https://doi.org/10.1016/j. semcancer.2017.04.008)

Fortner RT, Schock H, Le Cornet C, Husing A, Vitonis AF, Johnson TS, Fichorova RN, Fashemi T, Yamamoto HS, Tjønneland A, et al. 2018 Ovarian cancer early detection by circulating CA125 in the context of anti-CA125 autoantibody levels: results from the EPIC cohort. International Journal of Cancer 142 1355-1360. (https://doi. org/10.1002/ijc.31164)

Gadducci A, Cosio S, Zola P, Landoni F, Maggino T \& Sartori E 2007 Surveillance procedures for patients treated for epithelial ovarian cancer: a review of the literature. International Journal of Gynecological Cancer 17 21-31. (https://doi org/10.1111/j.1525-1438.2007.00826.x)

Gonzalez-Angulo AM, Iwamoto T, Liu S, Chen H, Do KA, Hortobagyi GN, Mills GB, Meric-Bernstam F, Symmans WF \& Pusztai L 2012 Gene expression, molecular class changes, and pathway analysis after neoadjuvant systemic therapy for breast cancer. Clinical Cancer Research 18 1109-1119. (https://doi. org/10.1158/1078-0432.CCR-11-2762)

Hanaoka T, Hasegawa K, Kato T, Sato S, Kurosaki A, Miyara A, Nagao S, Seki H, Yasuda M \& Fujiwara K 2017 Correlation between tumor mesothelin expression and serum mesothelin in patients with epithelial ovarian carcinoma: a potential noninvasive biomarker for mesothelin-targeted therapy. Molecular Diagnosis and Therapy 21 187-198. (https://doi.org/10.1007/s40291-017-0255-2)

Hiramatsu K, Yoshino K, Serada S, Yoshihara K, Hori Y, Fujimoto M, Matsuzaki S, Egawa-Takata T, Kobayashi E, Ueda Y, et al. 2016 Similar protein expression profiles of ovarian and endometrial high-grade
2018 Society for Endocrinology Published by Bioscientifica Ltd. Printed in Great Britain 
serous carcinomas. British Journal of Cancer 114 554-561. (https:// doi.org/10.1038/bjc.2016.27)

Hu R, Wang X \& Zhan X 2013 Multi-parameter systematic strategies for predictive, preventive and personalised medicine in cancer. EPMA Journal 4 2. (https://doi.org/10.1186/1878-5085-4-2)

Huhta H, Helminen O, Palomaki S, Kauppila JH, Saarnio J, Lehenkari PP \& Karttunen TJ 2017 Intratumoral lactate metabolism in Barrett's esophagus and adenocarcinoma. Oncotarget 8 22894-22902. (https:// doi.org/10.18632/oncotarget.15284)

Jiang XS, Zhou H, Zhang L, Sheng QH, Li SJ, Li L, Hao P, Li YX, Xia QC, Wu JR, et al. 2004 A high-throughput approach for subcellular proteome: identification of rat liver proteins using subcellular fractionation coupled with two-dimensional liquid chromatography tandem mass spectrometry and bioinformatic analysis. Molecular and Cellular Proteomics 3 441-455. (https://doi.org/10.1074/mcp. M300117-MCP200)

Jiang Y, Yan F, Liang L, Wan Y, Liu J \& Cheng W 2017 Meta-analysis demonstrates no association between p16 (ink4a) promoter methylation and epithelial ovarian cancer. Archives of Gynecology and Obstetrics 295 697-704. (https://doi.org/10.1007/s00404-016-4264-x)

Karabudak AA, Hafner J, Shetty V, Chen S, Secord AA, Morse MA \& Philip R 2013 Autoantibody biomarkers identified by proteomics methods distinguish ovarian cancer from non-ovarian cancer with various CA-125 levels. Journal of Cancer Research and Clinical Oncology 139 1757-1770. (https://doi.org/10.1007/s00432-013-1501-6)

Khan A, Eikani CK, Khan H, Iavarone AT \& Pesavento JJ 2018 Characterization of Chlamydomonas reinhardtii core histones by top-down mass spectrometry reveals unique algae-specific variants and post-translational modifications. Journal of Proteome Research 17 23-32. (https://doi.org/10.1021/acs.jproteome.7b00780)

Kim TW, Kim B, Kim JH, Kang S, Park SB, Jeong G, Kang HS \& Kim SJ 2013 Nuclear-encoded mitochondrial MTO1 and MRPL41 are regulated in an opposite epigenetic mode based on estrogen receptor status in breast cancer. BMC Cancer 13 502. (https://doi. org/10.1186/1471-2407-13-502)

Kim HK, Noh YH, Nilius B, Ko KS, Rhee BD, Kim N \& Han J 2017 Current and upcoming mitochondrial targets for cancer therapy. Seminars in Cancer Biology 47 154-167. (https://doi.org/10.1016/j. semcancer.2017.06.006)

Ksiezakowska-Lakoma K, Kulczycka-Wojdala D, Kulig A, Baum M \& Wilczynski JR 2017 The presence of A5935G, G5949A, G6081A, G6267A, T9540C mutations in MT-CO1 and MT-CO3 genes and other variants of MT-CO1 and MT-CO3 gene fragments in the study population diagnosed with endometrial cancer. Ginekologia Polska $\mathbf{8 8}$ 343-348. (https://doi.org/10.5603/GP.a2017.0065)

Lemasters JJ 2005 Selective mitochondrial autophagy, or mitophagy, as a targeted defense against oxidative stress, mitochondrial dysfunction, and aging. Rejuvenation Research 8 3-5. (https://doi.org/10.1089/ rej.2005.8.3)

Michalak S, Rybacka-Mossakowska J, Gazdulska J, Golda-Gocka I \& Ramlau R 2016 The effect on cognition of mitochondrial respiratory system proteins in peripheral blood mononuclear cells in the course of lung cancer. Advances in Experimental Medicine and Biology 911 45-52. (https://doi.org/10.1007/5584_2016_221)

Miller KD, Siegel RL, Lin CC, Mariotto AB, Kramer JL, Rowland JH, Stein KD, Alteri R \& Jemal A 2016 Cancer treatment and survivorship statistics, 2016. CA: A Cancer Journal for Clinicians 66 271-289. (https://doi.org/10.3322/caac.21349)

Pasing Y, Schniers A \& Hansen T 2017 Straightforward protocol for gelfree proteomic analysis of adipose tissue. Methods in Molecular Biology [epub]. (https://doi.org/10.1007/7651_2017_82)

Peng F, Li J, Guo T, Yang H, Li M, Sang S, Li X, Desiderio DM \& Zhan X 2015 Nitroproteins in human astrocytomas discovered by gel electrophoresis and tandem mass spectrometry. Journal of the American Society for Mass Spectrometry 26 2062-2076. (https://doi. org/10.1007/s13361-015-1270-3)
Pu M, Wang J, Huang Q, Zhao G, Xia C, Shang R, Zhang Z, Bian Z, Yang X \& Tao K 2017 High MRPS23 expression contributes to hepatocellular carcinoma proliferation and indicates poor survival outcomes. Tumor Biology 39 1393380537. (https://doi. org/10.1177/1010428317709127)

Qi Y, Xu F, Chen L, Li Y, Xu Z, Zhang Y, Wei W, Su N, Zhang T, Fan F, et al. 2016 Quantitative proteomics reveals FLNC as a potential progression marker for the development of hepatocellular carcinoma. Oncotarget 7 68242-68252. (https://doi.org/10.18632/ oncotarget.11921)

Ray S, Reddy PJ, Jain R, Gollapalli K, Moiyadi A \& Srivastava S 2011 Proteomic technologies for the identification of disease biomarkers in serum: advances and challenges ahead. Proteomics 11 2139-2161. (https://doi.org/10.1002/pmic.201000460)

Riley NM \& Coon JJ 2018 The role of electron transfer dissociation in modern proteomics. Analytical Chemistry 90 40-64. (https://doi. org/10.1021/acs.analchem.7b04810)

Sakhuja S, Yun H, Pisu M \& Akinyemiju T 2017 Availability of healthcare resources and epithelial ovarian cancer stage of diagnosis and mortality among Blacks and Whites. Journal of Ovarian Research 10 57. (https://doi.org/10.1186/s13048-017-0352-1)

Schonenberger MJ \& Kovacs WJ 2017 Isolation of peroxisomes from mouse brain using a continuous nycodenz gradient: a comparison to the isolation of liver and kidney peroxisomes. Methods in Molecular Biology 1595 13-26. (https://doi.org/10.1007/978-1-4939. 6937-1_2)

Simon I, Katsaros D, Rigault DLLI, Massobrio M, Scorilas A, Kim NW, Sarno MJ, Wolfert RL \& Diamandis EP 2007 B7-H4 is over-expressed in early-stage ovarian cancer and is independent of CA125 expression. Gynecologic Oncology 106 334-341. (https://doi. org/10.1016/j.ygyno.2007.03.035)

Sloutsky R \& Naegle KM 2018 Proteome-level analysis indicates global mechanisms for post-translational regulation of RRM domains. Journal of Molecular Biology 430 41-44. (https://doi.org/10.1016/j. jmb.2017.11.001)

Sotgia F, Whitaker-Menezes D, Martinez-Outschoorn UE, Salem AF, Tsirigos A, Lamb R, Sneddon S, Hulit J, Howell A \& Lisanti MP 2012 Mitochondria "fuel" breast cancer metabolism: fifteen markers of mitochondrial biogenesis label epithelial cancer cells, but are excluded from adjacent stromal cells. Cell Cycle $114390-4401$. (https://doi.org/10.4161/cc.22777)

Stewart ML, Tamayo P, Wilson AJ, Wang S, Chang YM, Kim JW, Khabele D, Shamji AF \& Schreiber SL 2015 KRAS genomic status predicts the sensitivity of ovarian cancer cells to decitabine. Cancer Research 75 2897-2906. (https://doi.org/10.1158/0008-5472.CAN-142860)

Scott JG, Berglund A, Schell MJ, Mihaylov I, Fulp WJ, Yue B, Welsh E, Caudell JJ, Ahmed K, Strom TS, et al. 2017 A genome-based model for adjusting radiotherapy dose (GARD): a retrospective, cohortbased study. Lancet Oncology 18 202-211. (https://doi.org/10.1016/ S1470-2045(16)30648-9)

van der Wal L, Bezstarosti K, Sap KA, Dekkers D, Rijkers E, Mientjes E, Elgersma Y \& Demmers JAA 2018 Improvement of ubiquitylation site detection by Orbitrap mass spectrometry. Journal of Proteomics 172 49-56. (https://doi.org/10.1016/j.jprot.2017.10.014)

Wanders RJ \& Waterham HR 2006 Biochemistry of mammalian peroxisomes revisited. Annual Review of Biochemistry 75 295-332. (https://doi.org/10.1146/annurev.biochem.74.082803.133329)

Wang X, Guo T, Peng F, Long Y, Mu Y, Yang H, Ye N, Li X \& Zhan X 2015 Proteomic and functional profiles of a follicle-stimulating hormone positive human nonfunctional pituitary adenoma. Electrophoresis 36 1289-1304. (https://doi.org/10.1002/ elps.201500006)

Wu H, Wei H, Sehgal SA, Liu L \& Chen Q 2016 Mitophagy receptors sense stress signals and couple mitochondrial dynamic machinery for mitochondrial quality control. Free Radical Biology and Medicine 
100 199-209. (https://doi.org/10.1016/j.

freeradbiomed.2016.03.030)

Zhan X, Long Y, Zhan X \& Mu Y 2017a Consideration of statistical vs. biological significances for omics data-based pathway network analysis. Med One 2 e170002. (https://doi.org/10.20900/ mo.20170002)

Zhan X, Long Y \& Lu M $2017 b$ Exploration of variations in proteome and metabolome for predictive diagnostics and personalized treatment algorithms: innovative approach and examples for potential clinical application. Journal of Proteomics [epub]. (https:// doi.org/10.1016/j.jprot.2017.08.020)

Zhan X, Qian S \& Huang Y 2017c The untapped potential of nitroproteomics for medicine. Med One 2 e170027. (https://doi. org/10.20900/mo.20170027)

Zhan X, Yang H, Peng F, Li J, Mu Y, Long Y, Cheng T, Huang Y, Li Z, Lu M, et al. 2018 How many proteins can be identified in a 2-DE gel spot within an analysis of a complex human cancer tissue proteome? Electrophoresis 39 965-980. (https://doi.org/10.1002/elps.201700330)
Zhang LL, Shao SL \& Wu Y 2010 Expressions of osteopontin and B7-H4 in epithelial ovarian neoplasm and their significance. Chinese Journal of Cancer 29 25-29. (https://doi.org/1000-467X(2010)01-0025-05)

Zhang H, Meltzer P \& Davis S 2013 RCircos: an R package for Circos 2D track plots. BMC Bioinformatics 14244 . (https://doi. org/10.1186/1471-2105-14-244)

Zhang H, Liu T, Zhang Z, Payne SH, Zhang B, McDermott JE, Zhou JY, Petyuk VA, Chen L, Ray D, et al. 2016 Integrated proteogenomic characterization of human high-grade serous ovarian cancer. Cell 166 755-765. (https://doi.org/10.1016/j.cell.2016.05.069)

Zhou JY, Chen L, Zhang B, Tian Y, Liu T, Thomas SN, Chen L, Schnaubelt M, Boja E, Hiltke T, et al. 2017 Quality assessments of long-term quantitative proteomic analysis of breast cancer xenograft tissues. Journal of Proteome Research 16 4523-4530. (https://doi. org/10.1021/acs.jproteome.7b00362)

Zimmermann M \& Reichert AS 2017 How to get rid of mitochondria: crosstalk and regulation of multiple mitophagy pathways. Biological Chemistry 399 29-45. (https://doi.org/10.1515/hsz-2017-0206)

Received in final form 12 June 2018

Accepted 19 June 2018 http://erc.endocrinology-journals.org https://doi.org/10.1530/ERC-18-0243
(C) 2018 Society for Endocrinology Published by Bioscientifica Ltd. Printed in Great Britain 